\title{
AKT-mediated stabilization of histone methyltransferase WHSC1 promotes prostate cancer metastasis
}

\author{
Ni Li, ${ }^{1}$ Wei Xue, ${ }^{2}$ Huairui Yuan, ${ }^{1}$ Baijun Dong, ${ }^{2}$ Yufeng Ding, ${ }^{1}$ Yongfeng Liu, ${ }^{1}$ Min Jiang, ${ }^{1}$ Shan Kan, ${ }^{1}$ Tongyu Sun, ${ }^{1}$ Jiale Ren, ${ }^{3}$ \\ Qiang Pan, ${ }^{3}$ Xiang Li, ${ }^{1}$ Peiyuan Zhang, ${ }^{4}$ Guohong Hu, ${ }^{4}$ Yan Wang, ${ }^{5}$ Xiaoming Wang, ${ }^{6}$ Qintong Li, ${ }^{7}$ and Jun Qin ${ }^{1}$ \\ 'Key Laboratory of Stem Cell Biology, Chinese Academy of Sciences Center for Excellence in Molecular Cell Science, Institute of Health Sciences, Shanghai Institutes for Biological Sciences, Chinese Academy \\ of Sciences and Shanghai Jiao Tong University School of Medicine, University of Chinese Academy of Sciences, Shanghai, China. 'Department of Urology, Ren Ji Hospital, Shanghai Jiao Tong University, \\ Shanghai, China. ${ }^{3}$ Institute of Health Sciences, Shanghai Jiao Tong University School of Medicine and Shanghai Institutes for Biological Sciences, Chinese Academy of Sciences, Shanghai, China. ${ }^{4}$ Institute of \\ Health Sciences, Shanghai Institutes for Biological Sciences, Chinese Academy of Sciences and Shanghai Jiao Tong University School of Medicine, University of Chinese Academy of Sciences, Shanghai, China. \\ IInstitute of Pathology and Southwest Cancer Center, Southwest Hospital, Third Military Medical University, Chongqing, China. ${ }^{6}$ Department of Immunology, Nanjing Medical University, Nanjing, China. ${ }^{\top}$ West \\ China Second University Hospital, Key Laboratory of Birth Defects and Related Diseases of Women and Children, Ministry of Education, Sichuan University, Chengdu, China.
}

\begin{abstract}
Loss of phosphatase and tensin homolog (PTEN) and activation of the PI3K/AKT signaling pathway are hallmarks of prostate cancer (PCa). However, these alterations alone are insufficient for cells to acquire metastatic traits. Here, we have shown that the histone dimethyl transferase WHSC1 critically drives indolent PTEN-null tumors to become metastatic PCa. In a PTEN-null murine PCa model, WHSC1 overexpression in prostate epithelium cooperated with Pten deletion to produce a metastasis-prone tumor. Conversely, genetic ablation of Whsc1 prevented tumor progression in PTEN-null mice. Molecular characterization revealed that increased AKT activity due to PTEN loss directly phosphorylates WHSC1 at S172, preventing WHSC1 degradation by CRL4 ${ }^{\text {Cdt2 }}$ E3 ligase. Increased WHSC1 expression transcriptionally upregulates expression of RICTOR, a pivotal component of mTOR complex 2 (mTORC2), to further enhance AKT activity. Therefore, the AKT/WHSC1/mTORC2 signaling cascade represents a vicious feedback loop that elicits unrestrained AKT signaling. Furthermore, we determined that WHSC1 positively regulates Rac1 transcription to increase tumor cell motility. The biological importance of a WHSC1-mediated signaling cascade is substantiated by patient sample analysis in which WHSC1 signaling is tightly correlated with disease progression and recurrence. Taken together, our findings highlight a pivotal link between an epigenetic regulator, WHSC1, and key intracellular signaling molecules, AKT, RICTOR, and Rac1, to drive PCa metastasis.
\end{abstract}

\section{Introduction}

Prostate tumors are the most frequently diagnosed cancer in men worldwide, and they proceed through a series of defined states, including prostatic intraepithelial neoplasia (PIN) and adenocarcinoma, followed by progression to invasive and metastatic cancer $(1,2)$. Despite recent progress, the major clinical challenges are to provide an effective means to stratify metastatic cancer from indolent tumors and to treat patients who have a deadly metastatic cancer (3). Thus, understanding the underlying mechanism by which indolent lesions give rise to metastatic cancer will likely benefit disease diagnosis and intervention.

Recurrent mutations, copy number alterations, and chromosomal rearrangements are implicated in prostate cancer (PCa) progression and metastasis (4-7). Of metastatic tumors, up to $70 \%$ exhibit phosphatase and tensin homolog (PTEN) loss-of-function mutations or genomic alterations in components of the PI3K signaling pathway $(4,8)$, indicating critical roles of PTEN and PI3K/ AKT signaling in $\mathrm{PCa}$ metastasis. Intriguingly, prostate-specific

Authorship note: N. Li, W. Xue, and H. Yuan contributed equally to this work. Conflict of interest: The authors have declared that no conflict of interest exists. Submitted: October 10, 2016; Accepted: January 19, 2017.

Reference information: / Clin Invest. 2017;127(4):1284-1302.

https://doi.org/10.1172/JCI91144.
Pten deletion in mice induces high-grade PIN (HGPIN) or adenocarcinoma, but with minimally invasive carcinoma incidence $(8,9)$. Previous studies have indicated that PTEN loss triggers a defense mechanism partially through feedback activation of TGF- $\beta$ and p53 signaling to constrain tumor malignancy $(9,10)$. Likewise, disruptions of such restricted barriers lead to full-blown disease (9-11). In addition, compelling evidence indicates that signaling circuit alterations, such as overexpression of ETS-related gene (ERG) and MAPK or downregulation of NK3 homeobox 1 (NKX3.1) and the $\mathrm{PH}$ domain and leucine-rich repeat protein phosphatase 1 (PHLPP1), facilitate the metastatic transformation of PTEN-null indolent tumors (12-17). These observations underscore that a "second hit" is indispensable for the acquisition of metastatic traits during tumor cell evolution.

Epigenetic perturbations are emerging as important contributing factors for tumorigenesis (18). Among these factors, histone methyltransferases constitute a compelling target for anticancer therapy because their enzymatic activity can be feasibly manipulated (19). For instance, enhancer of zeste homolog 2 (EZH2), a subunit of Polycomb repressive complex 2 (PRC2), has been demonstrated to have a prominent function in tumorigenesis, and compounds that target EZH2 are undergoing clinical trials (20-23). Interestingly, one study showed that EZH2 transcriptionally upregulates the expression of Wolf-Hirschhorn syndrome 
candidate 1 (WHSC1) through negative regulation of microRNA levels and that the oncogenic functions of EZH2 largely depend on WHSC1 (24). WHSC1 (also known as MMSET and NSD2) is a histone methyltransferase that catalyzes the dimethylation of histone $\mathrm{H} 3$ at lysine 36 (H3K36me2), a permissive mark associated with active gene transcription $(25,26)$. WHSC1 haploinsufficiency is linked to Wolf-Hirschhorn syndrome (WHS) (27), which is manifested by growth and mental retardation as well as congenital heart defects. As supporting evidence, Whsc1 germline knockout mice displayed embryonic development defects reminiscent of WHS symptoms (28). Beyond the roles of WHSC1 in development, WHSC1 is also intimately associated with human tumorigenesis. The oncogenic role of WHSC1 was first reported in multiple myeloma, in which the $(4 ; 14)(\mathrm{p} 16 ; \mathrm{q} 32)$ translocation results in WHSC1 overexpression (29-31). WHSC1 is also frequently overexpressed in solid tumors such as oligodendroglioma, breast, prostate, and head and neck cancers (32-34). Cell culture studies indicated that WHSC1 modulates NIMA-related kinase-7 (NEK7), Twist family bHLH transcription factor 1 (TWIST1), and nuclear factor $\kappa$-light-chain-enhancer of activated B cells (NF- $\kappa \mathrm{B})$ to promote tumorigenesis (34-36). Nevertheless, the genetic characterization of WHSC1 to determine its role in PCa metastasis and the signaling coordinated by WHSC1 remain undefined.

Here, we use genetically engineered mouse (GEM) models and establish that WHSC1 is a critical determinant for full metastatic transformation of PTEN-null indolent tumors. Molecular characterization illustrates that AKT directly phosphorylates WHSC1-S172 to prevent its degradation by CRL4 $4^{\text {Cdt2 }}$ E3 ligase. This posttranscriptional regulation contributes to the effects of WHSC1 on PCa metastasis. More importantly, our study reveals that an unexpected but critical feedback loop between epigenetic (WHSC1) and key intracellular (PTEN or AKT/RICTOR/Rac1) molecules promotes full penetrance of metastatic $\mathrm{PCa}$.

\section{Results}

WHSC1 is associated with human metastatic PCa. In line with the increased expression of WHSC1 reported in a subset of human primary prostate tumors (24), our data mining using public data sets indicated that WHSC1 expression was elevated in tumors compared with normal counterparts (refs. 4, 37, 38; Figure 1A; and Supplemental Table 1; supplemental material available online with this article; https://doi.org/10.1172/JCI91144DS1). In addition, correlation studies demonstrated that WHSC1 expression was positively associated with adverse clinicopathological features, including seminal vesicle invasion and the Gleason score (Supplemental Figure 1). Interestingly, we noticed that metastatic tumors exhibited a more profound increase in WHSC1 expression among the different patient cohorts examined (Figure 1A). In a relevant study, a Kaplan-Meier plot revealed the significant association between higher WHSC1 expression and disease relapse in 2 separate patient cohorts (using data sets GSE21032 and GSE40272; Figure 1B and refs. 4, 39). To further assess the clinical relevance of WHSC1 in PCa, we performed IHC staining with a validated antibody against WHSC1 on an Asian radical prostatectomy cohort (40), a tumor tissue microarray (TMA) composed of 118 patient specimens (Supplemental Table 2). Examination of clinical prostate specimens showed significantly higher WHSC1 expres- sion in prostate tumors (mean 3.91, median 4.00) in comparison with the adjacent normal prostate counterparts (mean 1.43, median 1.52). As summarized in Figure 1C, approximately $50 \%$ of PCa specimens exhibited intermediate to intense nuclear WHSC1 staining, whereas less than $25 \%$ of normal prostate counterparts displayed such a pattern. More importantly, WHSC1 expression levels in prostate tumor cells behaved as an independent predictor to stratify the risk of biochemical recurrence in patients (Figure 1D). Cox proportional hazards regression analyses revealed that tumor recurrence was highly sensitive to a gradual increase in the WHSC1 protein level, as judged by the increasing hazard ratios (HRs). All together, these findings indicate that WHSC1 is a potential biomarker for PCa diagnosis and suggest that WHSC1 might play a causal role in PCa metastasis.

WHSC1 promotes the metastasis of PCa cells. To define the role of WHSC1 in PCa metastasis, we first generated WHSC1-depleted LNCaP and PC3 cells. In agreement with the previous findings $(33,35)$, silencing WHSC1 expression compromised the growth, migration, and anchorage-independent growth of PCa cells (Supplemental Figure 2, A-C and E). Conversely, overexpression of WHSC1 enhanced the migration of PCa cells, whereas a WHSC1 mutant lacking a catalytic SET domain abolished its oncogenic effects (Supplemental Figure 2, D and E). Next, we conducted an intracardiac injection assay to determine whether dysregulated WHSC1 expression altered tumor cell metastasis to the bone. A PC3-based cell line stably expressing the luciferase gene was used to visualize the metastatic lesion via luminescence, and WHSC1 was overexpressed or depleted, as shown in Figure 2A. As revealed by bioluminescent imaging (BLI), tumor cells bearing ectopically expressed WHSC1 (WHSC1-OE) profoundly metastasized to the skeleton after 1 month of inoculations, whereas control mice displayed significantly fewer luminescent signals (Figure 2A). During 2 months of follow-up, we observed that mice injected with WHSC1-OE displayed a greatly decreased median survival and had paralyzed limbs caused by severe bone lesions (Supplemental Figure 2F). As supporting evidence, there was a prominent increase in osteolysis in the WHSC1-OE group (Figure 2B). In sharp contrast, we noticed starkly decreased colonization of tumor cells within the skeleton and significantly longer lifespan in the absence of WHSC1 (Figure 2, A and B). This observation was strengthened by histopathological examination. We stained the bone lesions with tartrate-resistant acid phosphatase (TRAP), a hallmark of activated osteoclasts, to determine whether the lesions had fully progressed. Consistent with BLI, positive staining was markedly increased in WHSC1-OE mice (Figure 2C). Likewise, E-cadherin was used to trace the epithelial origin of $\mathrm{PCa}$ cells; there were more colonized tumor foci within the skeleton in WHSC1-OE mice, whereas a loss of WHSC1 significantly impaired the metastasis of PCa cells (Figure 2C).

WHSC1 deficiency prevents the progression of PTEN-null tumors in mice. Next, we aimed to use GEMs to define the role of WHSC1 in prostate tumorigenesis. We first generated $W h s c 1^{f l f l}$ mice to circumvent embryonic lethality (Supplemental Figure 3A) and crossed these mice with Probasin-Cre $\left(\mathrm{PB}^{\mathrm{Cre} /+}\right)$ mice to delete Whsc1 in the prostate epithelium (hereafter referred to as $\mathrm{WHSC1}^{\mathrm{PC}-/-}$ mice). Histopathological examination of prostates isolated from 4-month-old control and WHSC1 $1^{\mathrm{PC}-/-}$ mice indicated that WHSC1 
A

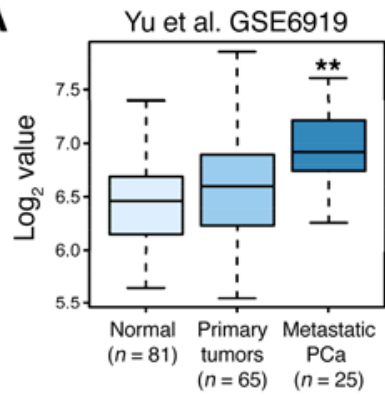

Taylor et al. GSE21032

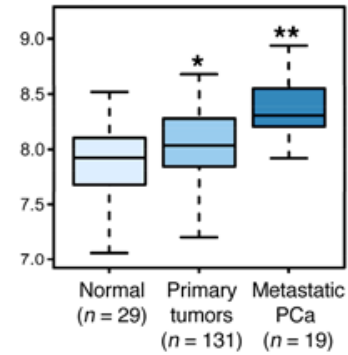

B
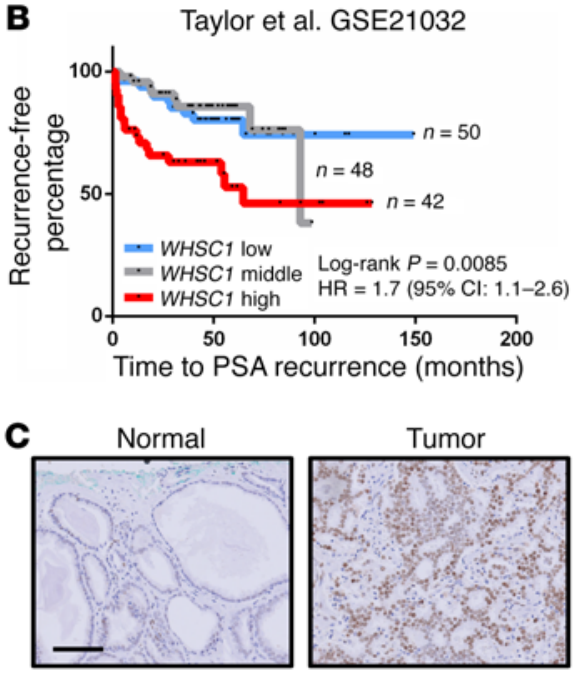

D

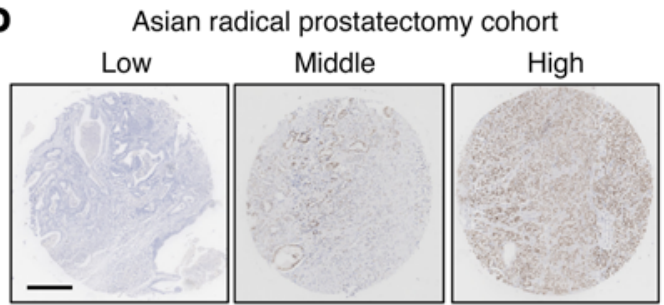

Gulzar et al. GSE40272
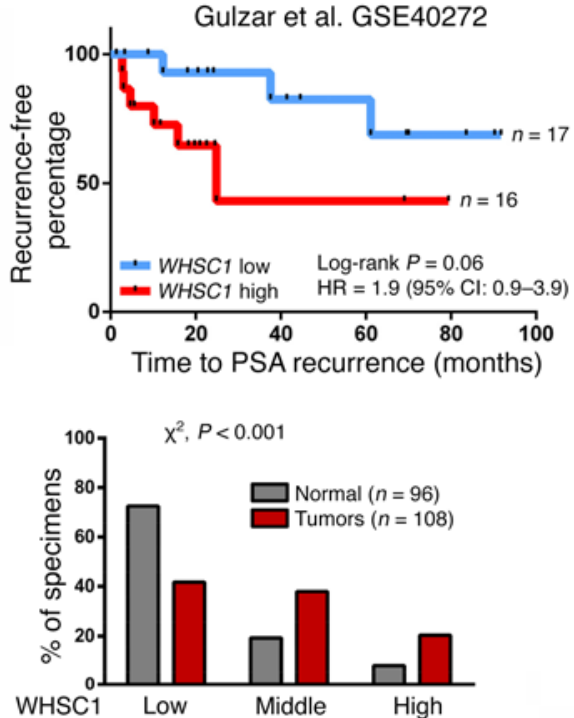

Figure 1. Clinical relevance of WHSC1 expression in PCa patients. (A) Box plot of WHSC1 expression levels in patients (Yu et al., GSE6919, ref. 37; Grasso et al., GSE35988, ref. 38; Taylor et al., GSE21032, ref. 4). Statistical significance is determined by 1-way ANOVA followed by Tukey's multiple comparisons test. ${ }^{*} P<0.05,{ }^{* *} P<0.01$. (B) The association of WHSC1 mRNA with biochemical recurrence (BCR) using data sets GSE21032 and GSE40272 ( $P$ values by log-rank test). (C) Expression and clinical relevance of WHSC1 were assessed in a human PCa TMA. WHSC1 staining indexes using a 10 -point quantification scale in cohorts of normal prostate counterparts $(n=96)$ and prostate tumors $(n=108)$ are shown. (D) Kaplan-Meier plot of recurrence after radical prostatectomy based on the WHSC1 expression index in patients ( $P$ values by log-rank test). HR, hazard ratio. Scale bars: $50 \mu \mathrm{m}$ (C), $300 \mu \mathrm{m}$ (D). was not essential for prostate development or homeostasis under physiological conditions (Supplemental Figure 3B). This observation was further strengthened by immunostaining for cytokeratin 8 (CK8) and transformation related protein 63 (p63) to indicate luminal epithelial cells and basal cells within the prostate epithelium, respectively (Supplemental Figure 3C). Overall, we did not detect any obvious differences between control and $\mathrm{WHSC1}^{\mathrm{PC}-/}$ mice under steady conditions.

Prostate-specific Pten deletion mice (Pten ${ }^{f / f l} \times \mathrm{PB}^{\mathrm{Cr} /+}$ mice, hereafter referred to as $\mathrm{PTEN}^{\mathrm{PC}-/-}$ mice) are accepted as an excellent mouse model to recapitulate tumor progression and histopathological features of the human disease $(4,8)$. In line with the notion that WHSC1 expression is increased in PCa patients, WHSC1 protein was significantly upregulated in $\mathrm{PTEN}^{\mathrm{PC}-/-}$ mice compared with WT controls (Figure 3A, top). Thus, we sought to determine whether WHSC1 is required for the progression of PTEN-null tumors. We crossed WHSC1 ${ }^{\mathrm{PC}-/-}$ mice with Pten $^{\text {fl/fl }}$ mice to ablate WHSC1 in a PTEN-null background (referred to hereaf- ter as $\mathrm{PTEN}^{\mathrm{PC}-/-} \mathrm{WHSC1}^{\mathrm{PC}-/-}$ mice; Figure $\left.3 \mathrm{~A}\right)$. The histopathological analysis of the entire animal cohorts revealed that depletion of WHSC1 compromised prostate tumor progression. As indicated in Figure 3B, PTEN ${ }^{\mathrm{PC}-/-}$ mice largely advanced to HGPIN or adenocarcinoma at 4 months of age, with less frequent development into locally invasive carcinoma. In sharp contrast, WHSC1-deficient mice developed lesions after a long latency, with incomplete penetrance, in which tumor progression was largely compromised at the low-grade PIN stage at 4 months of age (Figure 3B, bottom). Next, we examined whether WHSC1 inactivation compromised the proliferation advantage elicited by PTEN loss. The proliferative index, as measured by Ki-67-positive cells, was significantly reduced in the prostates of $\mathrm{PTEN}^{\mathrm{PC}-/-} \mathrm{WHSC1}^{\mathrm{PC}-/-}$ mice compared with those in $\mathrm{PTEN}^{\mathrm{PC}-/-}$ mice (Supplemental Figure 3D). Together, these results highlight that WHSC1 deficiency impairs the tumor progression induced by PTEN loss.

Overexpression of WHSC1 in prostate epithelium cooperates with PTEN deficiency to produce metastasis-prone PCa. Given that WHSC1 
A
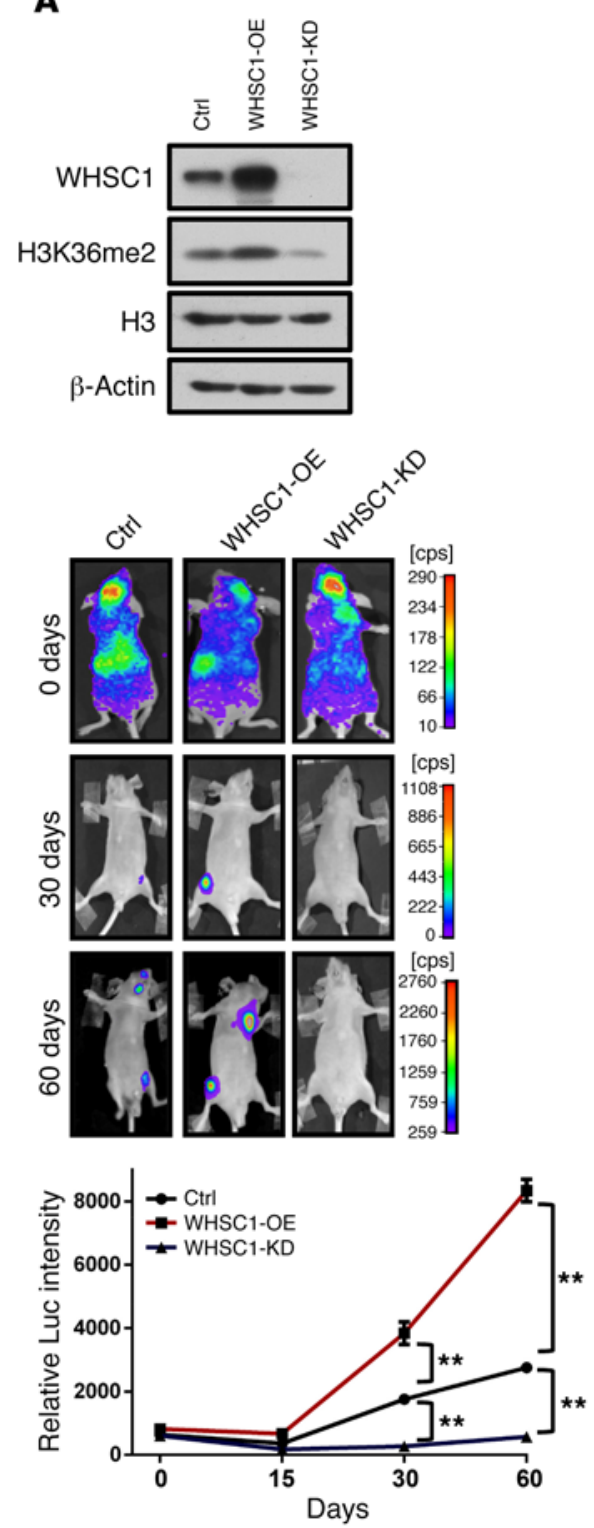

B
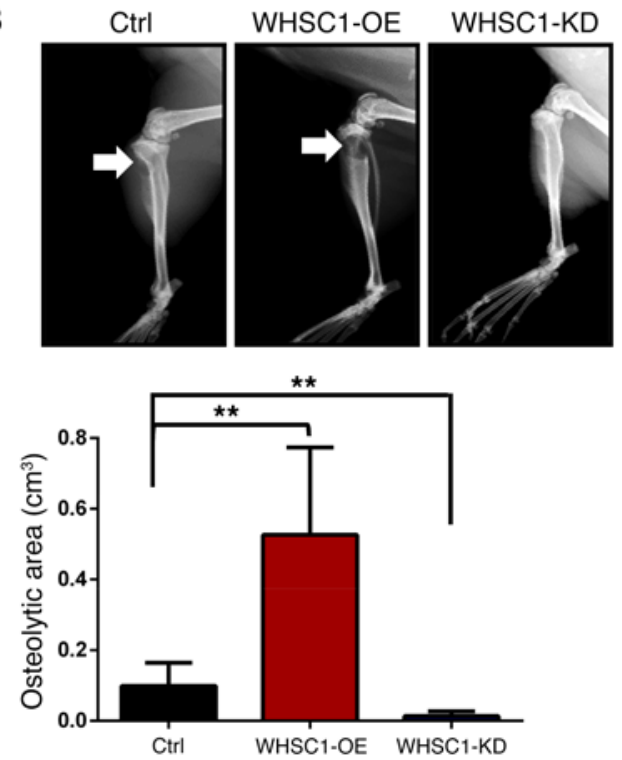

C
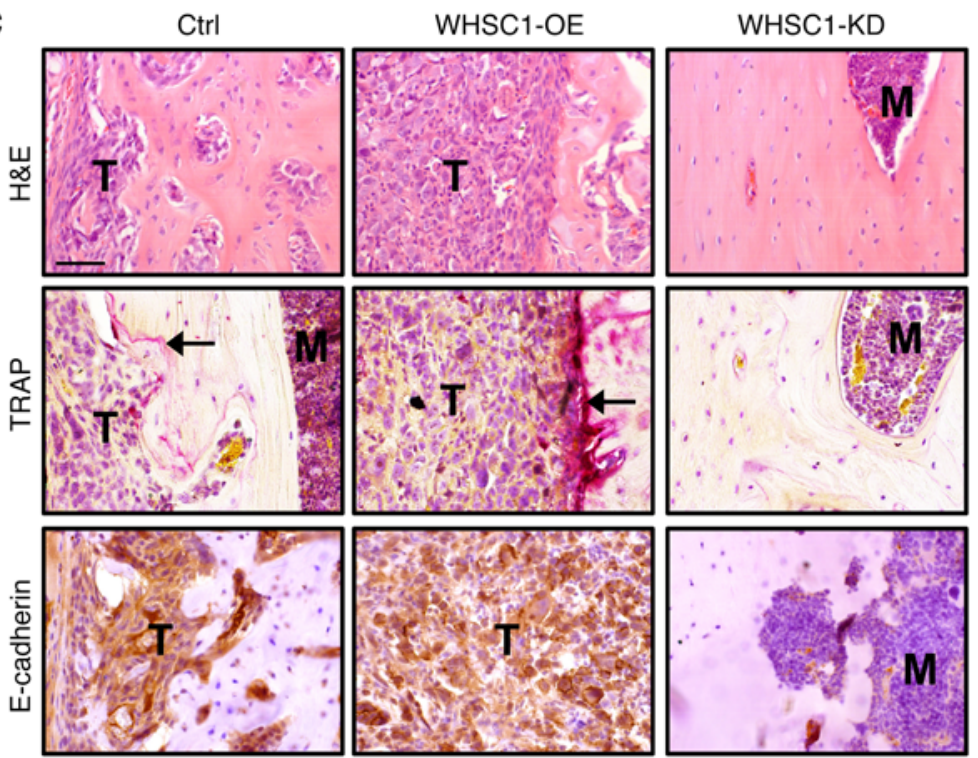

Figure 2. Ex vivo bone metastasis analyses of animals injected with WHSC1-depleted and WHSC1-overexpressing PC3 cells. (A) Western blot analysis of the indicated protein in control, WHSC1 overexpression (WHSC1-OE), and WHSC1 knockdown (WHSC1-KD) cells (top panel). The middle panel shows the representative BLI images for control, WHSC1-OE, and WHSC1-KD groups. BLI quantitation of limb metastasis, which was calculated as the means \pm SEM of bioluminescent signals, is shown in the bottom panel ( $n=9$ per group). One-way ANOVA followed by Tukey's multiple comparisons test, ${ }^{* *} P<0.01$. (B) Representative $x$-ray images for bone metastasis are shown in the top panel, and osteolytic area sizes are quantified in the bottom panel. One-way ANOVA followed by Tukey's multiple comparisons test, ${ }^{* *} P<0.01$. Arrows denote areas of overt osteolysis ( $n=8$ per group). (C) H\&E-, TRAP-, and E-cadherinstained images of bone sections from the mice as indicated. T, tumor cell; $\mathrm{M}$, bone marrow; arrow, TRAP-positive stained cells. Scale bar: $50 \mu \mathrm{m}$ (C).

expression is frequently upregulated in metastatic patients and correlates with disease relapse, we generated WHSC1 overexpression mice to mimic WHSC1 elevation in patients. WHSC1 ${ }^{\mathrm{OE} /+}$ mice harbor a single copy of a minigene consisting of a CAGGS (a hybrid chicken $\beta$-actin and cytomegalovirus) promoter, a loxP-STOP-loxP (LSL) cassette (a stop cassette consisting of 4 copies of polyadenylation sequences), and Myc-tagged WHSC1 cDNA knocked into the Rosa 26 locus (Figure $3 \mathrm{C}$ and Supplemental Figure 4A). In general, WHSC $1^{\mathrm{OE} /+}$ mice were healthy without overt defects in growth and reproduction. Upon crossing of these mice with $P B^{\mathrm{Cre} /+}$ mice, the LSL stop cassette was removed to produce WHSC1 overexpression within the prostate epithelium (WHSC1 ${ }^{\mathrm{PCOE} /+}$ ) (Supplemental Figure $4 \mathrm{~B}$, bottom). During 8 months of follow-up, $\mathrm{WHSC1}^{\mathrm{PCOE} /+}$ mice exhibited normal prostatic histology in a manner similar to that of control mice (Supplemental Figure 4B), indicating that cooperative oncogenic lesions might be required for WHSC1 functions.

Both PTEN loss and WHSC1 overexpression are frequently observed in metastatic PCa. Curiously, neither alone is sufficient to drive metastasis; therefore, we aimed to determine whether WHSC1 overexpression collaborates with PTEN loss to produce metastasis-prone cancer in mice. WHSC $1^{\mathrm{OE} /+}$ mice were crossed with $P$ ten ${ }^{f / f l}$ and $P B^{C r /+}$ mice to generate prostatic WHSC1 over- 
A

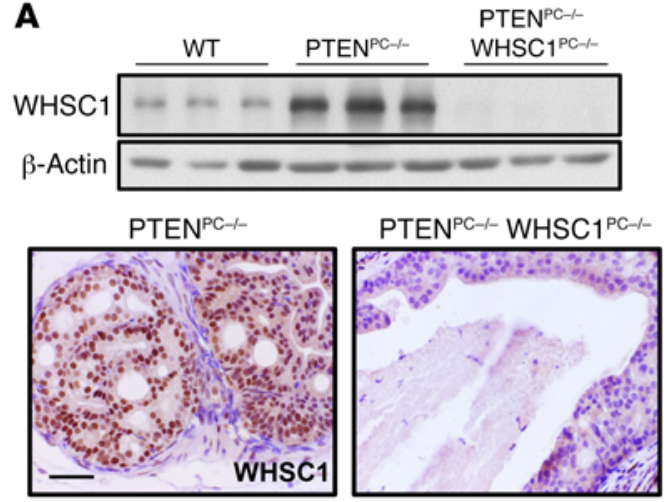

C

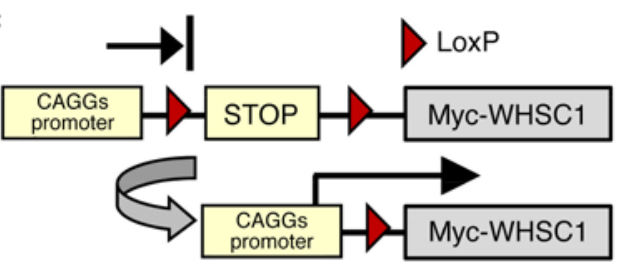

D

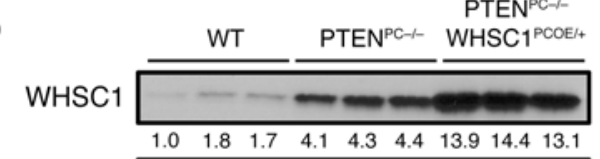

H3K36me2

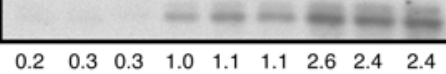

$\mathrm{H} 3$

$\beta$-Actin

\section{E}

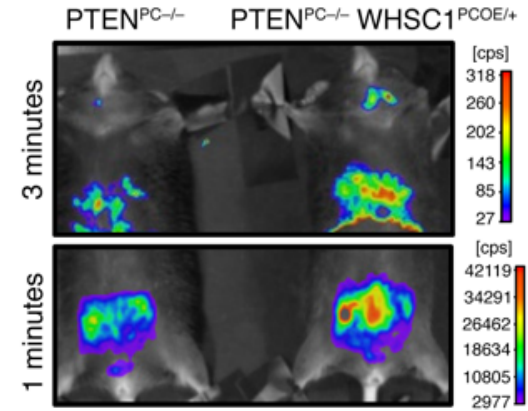

B

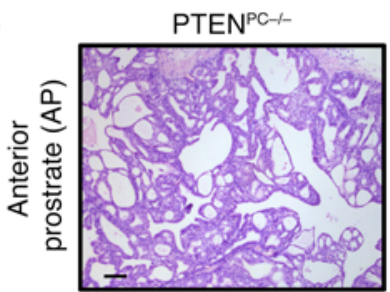

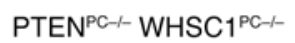
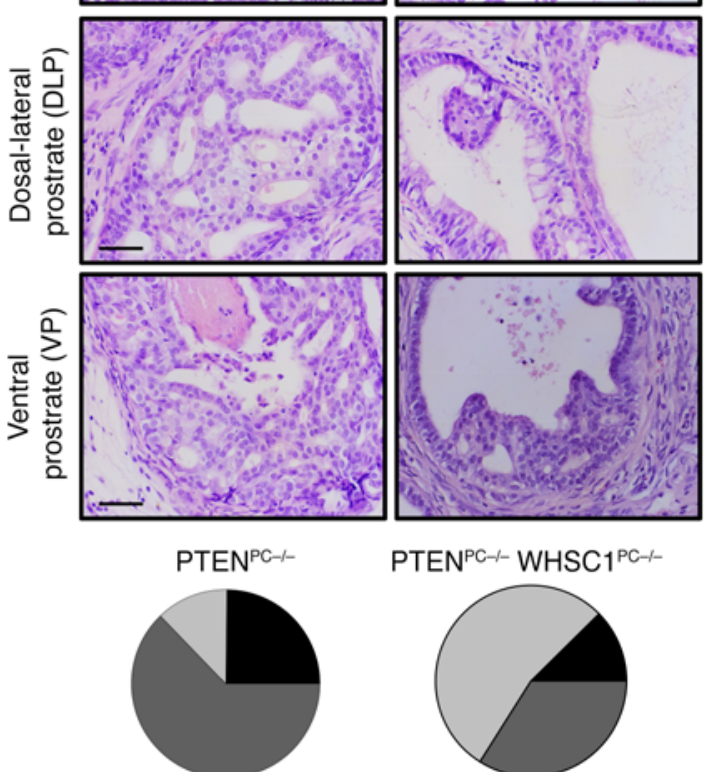

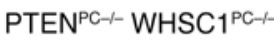
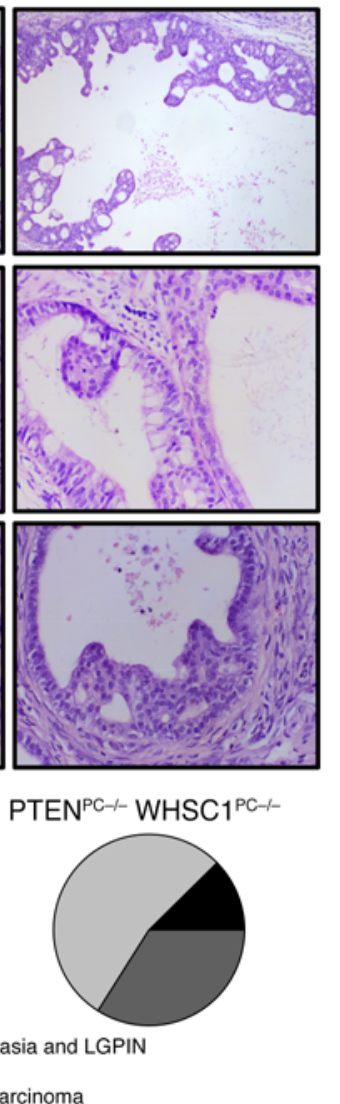

Hyperplasia and LGPIN

Adenocarcinoma
Lymph node metastasis

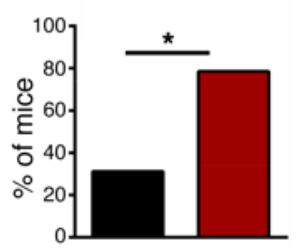

Lung metastasis

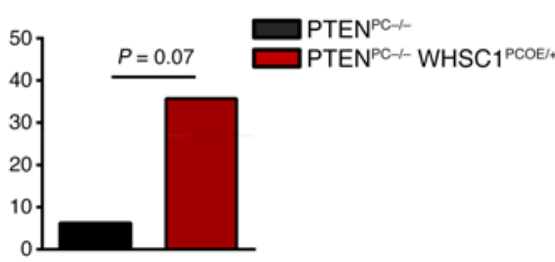

Figure 3. WHSC1 promotes PCa metastasis. (A and B) Inactivation of WHSC1 inhibits PTEN-deficient prostate tumor progression. (A) Western blot analysis of the indicated protein in anterior prostate (AP) lysates from 4-month-old WT, PTEN ${ }^{\mathrm{PC}-I_{-}}$, and $\mathrm{PTEN} \mathrm{PC}^{\mathrm{PC}-\mathrm{W}} \mathrm{WHSC}^{\mathrm{PC}-I_{-}}$mice (top). IHC staining for WHSC1 expression is shown in the bottom panel. (B) H\&E-stained sections of representative AP, dorsal-lateral prostate (DLP), and ventral prostate (VP) of 4-month-old mice. Pie graphs (bottom) show the results of tumor progression in PTEN ${ }^{\mathrm{PC}-{ }_{-}}$and PTEN ${ }^{\mathrm{PC}-I_{-}}$WHSC1PC-/- mice at 4 months of age. HCPIN, highgrade PIN; LGPIN, low-grade PIN. (C-E) Overexpression of WHSC1 cooperates with PTEN loss to develop metastatic PCa. (C) Scheme of WHSC1 ${ }^{0 E /+}$ mice.

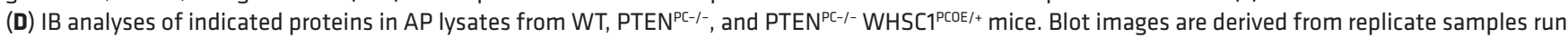
on parallel gels. (E) Representative luminescence images of PTEN ${ }^{\mathrm{PC}-I_{-}}$and $\mathrm{PTEN}^{\mathrm{PC}-I_{-}}$WHSC1 ${ }^{\mathrm{PCOE} /+}$ mice at 4 months of age. Quantification results of distal metastasis for the examined animals are shown in the right panel $(n>12)$. Fisher's exact test was used to determine statistical significance. ${ }^{*} P<0.05$. Scale bars: $50 \mu \mathrm{m}$ (A and $\mathbf{B})$.

expression in a PTEN-null background $\left(\mathrm{PTEN}^{\mathrm{PC}-/-}\right.$ WHSC1 ${ }^{\mathrm{PCOE} /+}$ mice). IB analysis indicated that WHSC1 expression was increased 3- to 4 -fold in the prostate epithelium of $\mathrm{PTEN}^{\mathrm{PC}-/-}$ WHSC1 ${ }^{\mathrm{PCOE} /+}$ mice in comparison with that of $\mathrm{PTEN}^{\mathrm{PC}-/}$ mice (Figure 3D). In line with the notion that WHSC1 catalyzes the dimethylation of histone $\mathrm{H} 3$ at lysine 36, we observed a simultaneous increase in H3K36me2 levels in WHSC1 overexpression mice. Notably, the expression level of WHSC1 in the overexpression mice was comparable with that in PCa cells, including LNCaP, LNCaP-Abl, and PC3 cells (Supplemental Figure $4 \mathrm{C}$ ), suggesting that the $\mathrm{PTEN}^{\mathrm{PC}-/-} \mathrm{WHSC1}^{\mathrm{PCOE} /+}$ mice used in this study could largely reflect the pathological conditions in human PCa. 
A

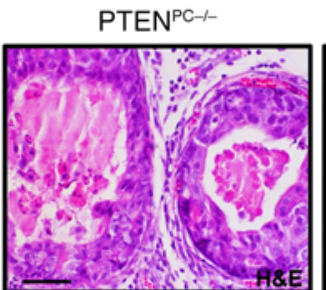

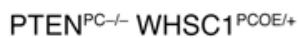
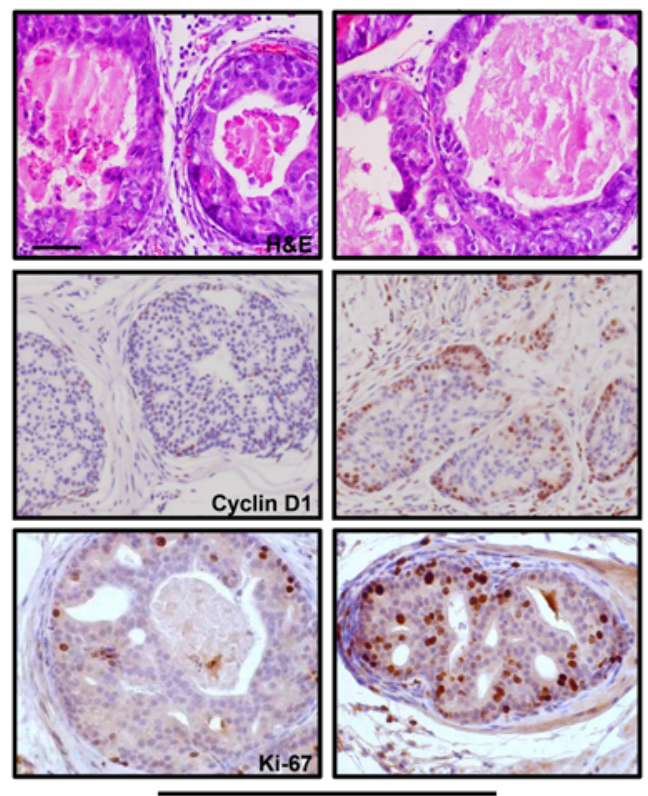

6 weeks

C
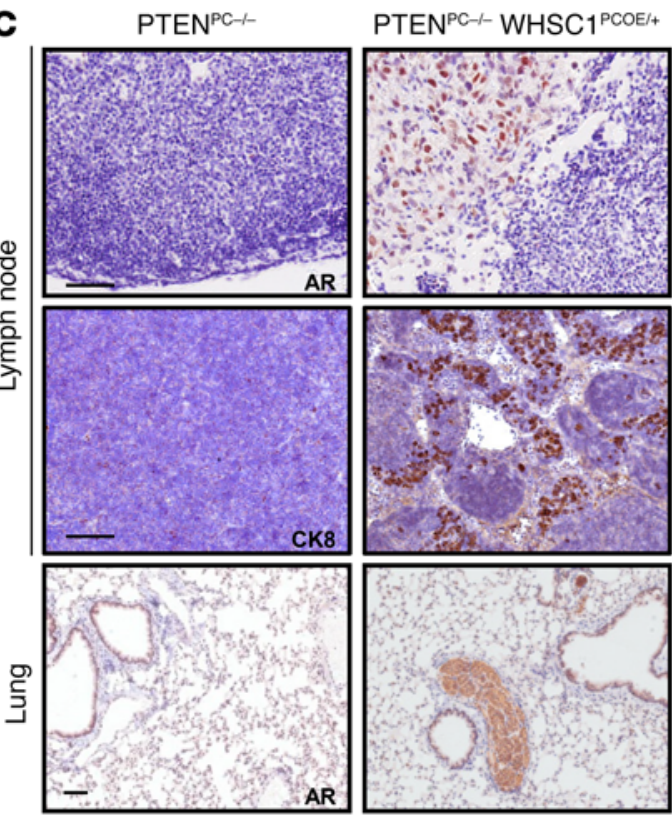

B

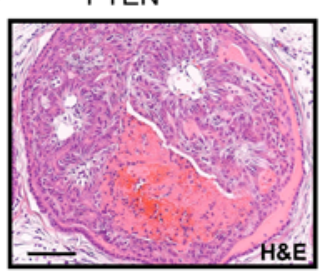

$\mathrm{PTEN}^{\mathrm{PC}-1-} \mathrm{WHSC}^{\mathrm{PCOE} /+}$
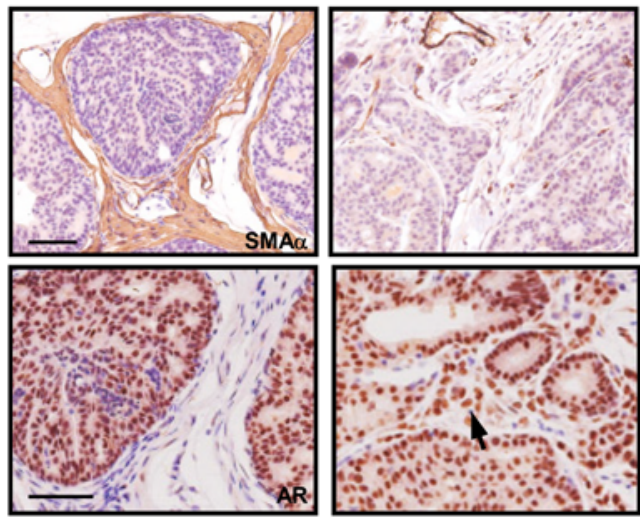

20 weeks
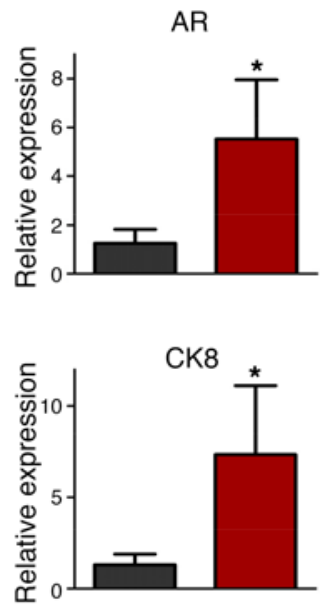

Figure 4. WHSC1 promotes PCa metastasis.

(A) H\&E-, cyclin D1-, and Ki-67-stained sections of representative DLP at 6 weeks of age. (B) H\&E-stained sections of DLP at 4 months of age (top). IHC analyses of aSMA (middle) and AR (bottom) in prostate tumors are shown. (C) IHC staining of AR and CK8 expression in lymph nodes and lungs from 4-month-old mice. Quantitative reverse transcriptase PCR (qRT-PCR) analysis of AR and CK8 expression in lymph nodes is shown in the right panel. Student's $t$ test, ${ }^{*} P<0.05$. Scale bars: $50 \mu \mathrm{m}(\mathbf{A}-\mathbf{C})$.

To better visualize tumor progression and metastasis in vivo, the compound mice were crossed with Rosa26-LSL-luciferase reporter mice for imaging analysis. As indicated in Figure 3E, $\mathrm{PTEN}^{\mathrm{PC}-/-}$ WHSC1 ${ }^{\mathrm{PCOE} /+}$ mice displayed much stronger bioluminescence intensity in the primary prostate lesions. More importantly, metastatic spreading of tumor cells to distant organs, including the lymph nodes and lungs, was observed with high penetrance in the WHSC1 overexpression mice. However, luminescent imaging analysis did not detect convincing bone metastatic lesions in WHSC1 overexpression mice. The quantitative results indicated that $\mathrm{PTEN}^{\mathrm{PC}-/-} \mathrm{WHSC1}^{\mathrm{PCOE} /+}$ mice harbored the metastatic spread of tumor nodules to lumbar lymph nodes in 11 of 14 cases and lung metastasis in 5 of 14 cases at 4 months of age (Figure $3 \mathrm{E}$, right).
This observation contrasted with $\mathrm{PTEN}^{\mathrm{PC}-/-}$ mice, in which 5 of 16 mice harbored lymph node metastasis, and only 1 of 16 mice developed metastatic lung lesions.

The results obtained from luminescent imaging analysis were further strengthened by histopathological analyses. At 6 weeks of age, both $\mathrm{PTEN}^{\mathrm{PC}-/-}$ and $\mathrm{PTEN}^{\mathrm{PC}-/-} \mathrm{WHSC1}^{\mathrm{PCOE} /+}$ mice developed HGPIN lesions without obvious morphological differences (Figure 4A, top). However, examination of the proliferation index, as indicated by positive cyclin D1 and Ki- 67 staining, revealed that tumor cells bearing a higher level of WHSC1 inherited advantageous growth traits (Figure 4A, middle and bottom). By 20 weeks of age, $\mathrm{PTEN}^{\mathrm{PC}-/-}$ WHSC1 ${ }^{\mathrm{PCOE} /+}$ mice developed focally invasive PCa with an altered stroma, as reflected by the attenuation or 



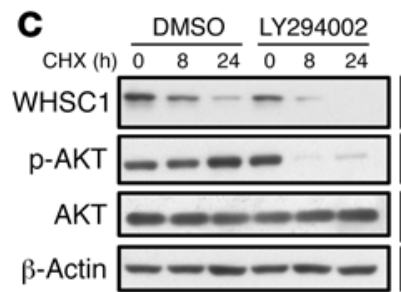
PC3

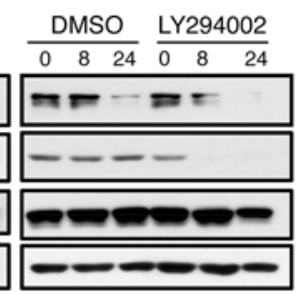

$\mathrm{LNCaP}$

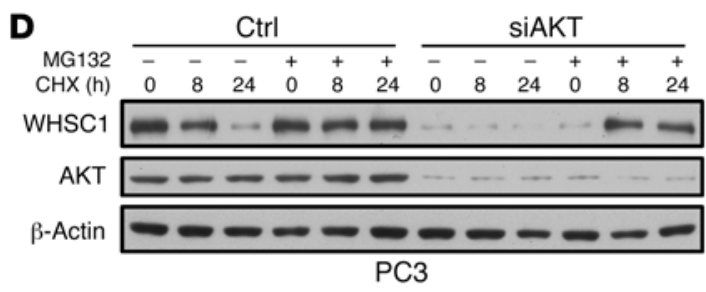

E RXRXXpS/pT

Homo sapiens ARRNRKRSIKYDSLL Mus mUSCUlus TRRNRKRSIKYDSLL Rattus norvegicus TRRNRKRSIKYDSLL Ovis aries ARRSRKRSAQRGSL Bos taurus ARRSRKRSAQRGPV Gallus gallus ERRNRKRSIKYDSLL

\section{F}

$\mathrm{IB}: \mathrm{AKT}=$

IB: WHSC1
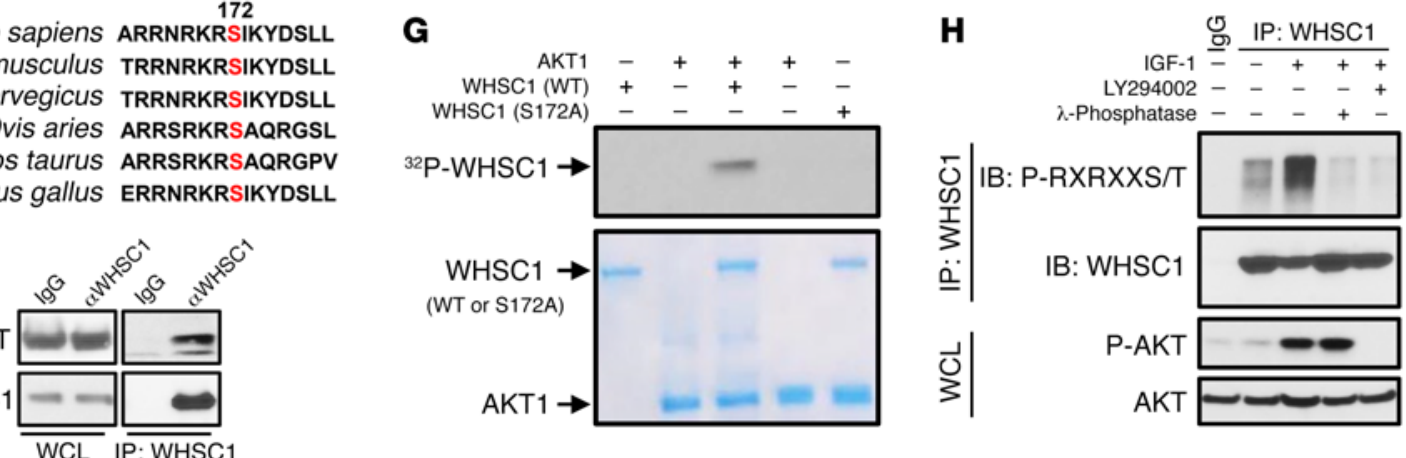

I

范

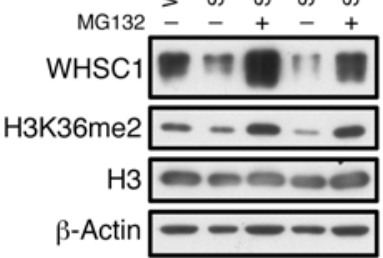

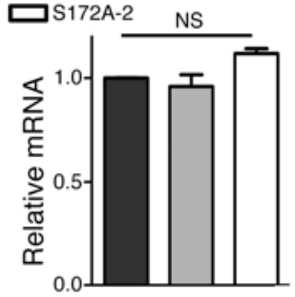

J

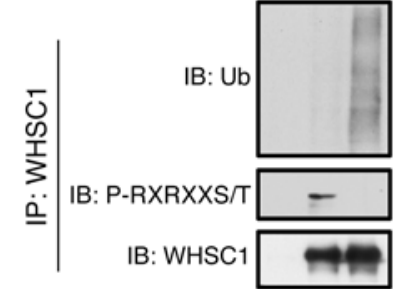

$\mathbf{K}$



S172A-1
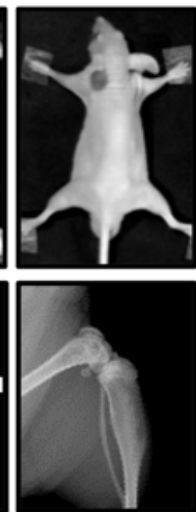

S172A-2
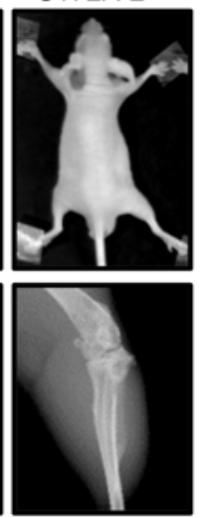
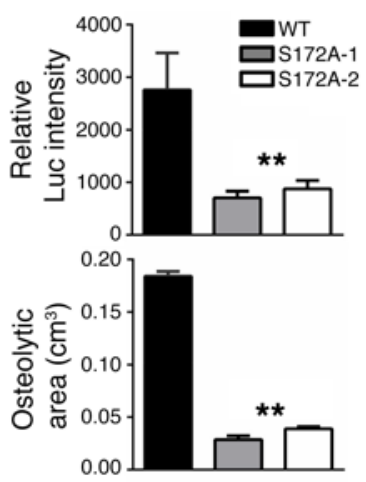

L
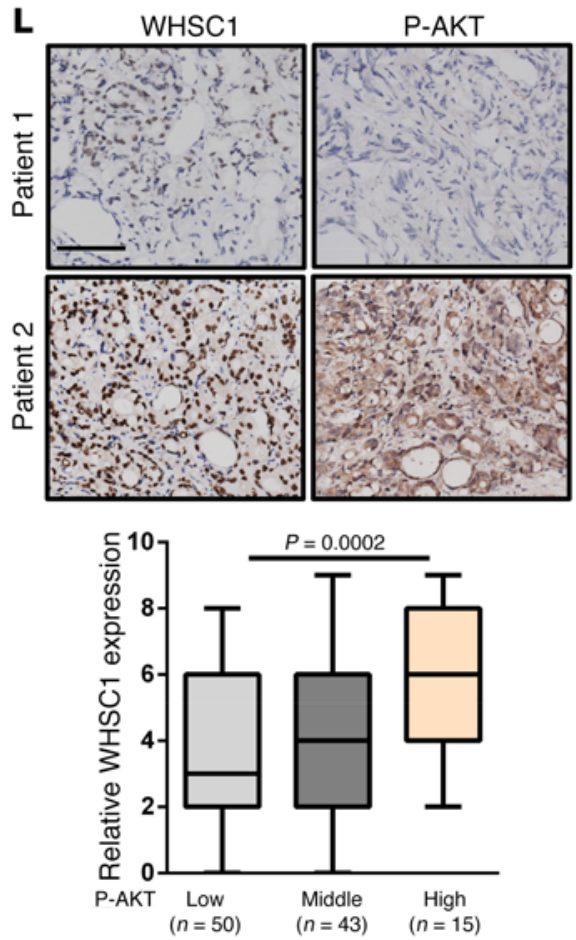
Figure 5. AKT phosphorylates WHSC1 at the S172 site to stabilize its protein in PCa cells. (A) Western blot analysis of the indicated protein in mouse AP lysates (mAP) and human prostate cell lines (left). qRT-PCR analysis of relative WHSC1 mRNA (right). (B) IB analysis of Pten ${ }^{f / f l}$ mouse embryonic fibroblasts (MEF) infected with Ad-GFP or Ad-Cre. Relative Pten and Whsc1 mRNA levels are shown in the right panel. Student's $t$ test, ${ }^{* *} P<0.01$. (C) IB analysis of PC3 and LNCaP cells treated with $30 \mu \mathrm{M}$ LY294002 for the indicated duration of time. (D) IB analysis of PC3 cells transfected with scramble or AKT oligonucleotides with or without MG132 treatment for the indicated duration of time. (E) Sequence alignment of the putative AKT phosphorylation site at S172 in WHSC1. (F) IB analysis of whole cell lysates (WCL) and immunoprecipitates derived from PC3 cells as indicated. (G) In vitro kinase assays depicting major AKT phosphorylation sites in WHSC1. (H) IB analysis of WCL and immunoprecipitates derived from PC3 cells treated with LY294002, IGF-1, or $\lambda$-phosphatase. (I) Left: The indicated protein in WT and S172A mutated cells. Right: Relative WHSC1 mRNA expression. Statistical significance was determined by 1-way ANOVA. (J) WT and S172A cell lysates were subjected to IP with anti-WHSC1 antibody and IB with anti-ubiquitin (anti-Ub) and anti-AKT substrate antibody. (K) Representative BLI and x-ray images of the mice after 2 months of inoculations with control and S172A cells (left). Quantitation of BLI and osteolytic area sizes in limbs (right; $n=5$ ). One-way ANOVA followed by Tukey's multiple comparisons test, ${ }^{* *} P<0.01$. (L) Representative IHC staining of WHSC1 and phospho-AKT in patients. Box plot shows the relative WHSC1 level in lower, middle, and higher phospho-AKT patients (an Asian radical prostatectomy cohort). Staining indexes using a 10 -point quantification scale. Low, $<4$; middle, 4 and 6 ; high, $>6$. Wilcoxon signed-rank test was used to calculate statistical significance. Scale bar: $50 \mu \mathrm{m}$ (L).

loss of the periglandular smooth muscle layer (Figure 4B). PTEN mutant tumors were largely indolent, characterized by wellconfined tumor cells within layers of aSMA-positive smooth muscle cells. In contrast, the smooth muscle sheaths surrounding the tumors in $\mathrm{PTEN}^{\mathrm{PC}-/-}$ WHSC1 ${ }^{\mathrm{PCOE} /+}$ mice were either discontinuous or partially disappeared in subset areas of prostatic acini. As a consequence, the luminal cell origins of prostate tumor cells indicated by androgen receptor-positive (AR-positive) cells extended beyond the basement membrane and invaded the stromal compartment (Figure 4B). The emergence of a potential epithelial-mesenchymal transition, as reflected by the reduction in E-cadherin and increase in vimentin expression, correlates with tumor invasion in comparison with the adjacent region with confined tumors (Supplemental Figure 4D). Since the WHSC1 overexpression allele was fused with a Myc tag, we used Myc positivity to track the epithelial-derived tumor cells and demonstrated that those invading cells are ectopically expressing vimentin, a mesenchymal marker (Supplemental Figure 4D). In line with the invasive tumors observed in primary tumors, molecular pathological analysis of PCa-bearing PTEN ${ }^{\mathrm{PC}-/-} \mathrm{WHSC1} 1^{\mathrm{PCOE} /+}$ mice showed metastatic spread of CK8- and AR-positive prostate tumor nodules in the lymph nodes and lungs (Figure 4C). Quantitative reverse transcriptase PCR analysis of $A r$ and keratin 8 transcripts revealed that there was more tumor invasion within the lymph nodes of $\mathrm{PTEN}^{\mathrm{PC}-/-} \mathrm{WHSC1}^{\mathrm{PCOE} /+}$ mice (Figure $4 \mathrm{C}$, right). Collectively, the GEM model indicates that WHSC1 overexpression cooperates with PTEN deficiency to promote PCa metastasis.

AKT directly phosphorylates WHSC1 at S172 to promote its stability. Having established the oncogenic role of WHSC1 in PCa metastasis, we aimed to determine which oncogenic insults led to WHSC1 elevation in metastatic PCa cells. Intriguingly, though the WHSC1 protein was increased in $\mathrm{PTEN}^{\mathrm{PC}-/-}$ mice (Figure 3A, top), we noticed that the Whsc1 mRNA did not display an appreciable difference between WT and PTEN ${ }^{\mathrm{PC}-/-}$ mice (Supplemental Figure 5A). Similarly, the WHSC1 protein (Figure 5A, left), but not its mRNA (right), was significantly increased in metastatic PCa cells (DU145, PC3, LNCaP, and LNCaP-Abl cells) compared with normal prostate epithelial cells (RWPE-1 cells and mouse anterior prostate), benign prostatic hyperplasia epithelial cells (BPH), and primary PCa cells (22RV-1) (Figure 5A). Likewise, depletion of PTEN in mouse embryonic fibroblasts and RWPE-1 cells led to a significant increase in the WHSC1 protein but not its mRNA (Figure 5B and Supplemental Figure 5B). These observations suggest that WHSC1 expression is upregulated by a posttranscriptional regulation mechanism.

In the context of PTEN loss, the most pronounced signaling event is the constitutive activation of AKT $(41,42)$, and it is known that AKT can influence proteasome-dependent protein degradation (43-45). Indeed, we noticed that the WHSC1 protein level appeared to be positively correlated with AKT activity in a subset of human PCa cells (Figure 5A, left), which prompted us to investigate whether there was a relationship between AKT activation and the WHSC1 protein level. When AKT signaling was inhibited by either LY294002 treatment (PI3K inhibitor) or siRNA knockdown, the half-life of the WHSC1 protein was attenuated (Figure 5, C and D). In addition, we showed that MG132 treatment reversed the effect of AKT depletion, suggesting that AKT prevents WHSC1 degradation in a proteasome-dependent manner (Figure 5D).

Next, we investigated how AKT regulated WHSC1 protein stability. There was a putative AKT phosphorylation motif RxRxxpS/ pT surrounding serine 172 in WHSC1, and this motif was evolutionarily conserved (Figure 5E). We therefore reasoned that WHSC1 might be a substrate of AKT. Indeed, mass spectrometry analyses revealed that the S172 site of WHSC1 was phosphorylated in PC3 cells (Supplemental Figure 5C). In addition, coimmunoprecipitation revealed that both ectopic and endogenous WHSC1 interacted with AKT in cells (Figure 5F and Supplemental Figure 5D). To examine whether AKT directly phosphorylates WHSC1, we performed in vitro kinase assays and showed that WT but not S172A mutant WHSC1 protein was directly phosphorylated by AKT (Figure $5 G$ ). In addition, WHSC1 phosphorylation was detected by the AKT substrate antibody. The intensity of phosphorylation was enhanced by IGF-1 and reduced by LY294002 treatment (Figure 5H and Supplemental Figure 5E). Notably, the reactivity of WHSC1 with AKT substrate antibody was eliminated by treatment with $\lambda$-phosphatase (Figure $5 \mathrm{H}$ ) or by preincubation of phospho-S172 peptide with the antibody (Supplemental Figure $5 F$ ), verifying the specificity of the detected WHSC1-S172 phosphorylation. Taken together, these results demonstrate that AKT directly phosphorylates $\mathrm{S} 172$ of WHSC1.

To define the biological significance of $\mathrm{S} 172$ phosphorylation, we substituted alanine for endogenous S172 in PC3 cells using the CRISPR/Cas9 technology (S172A cells; Supplemental Figure $5 G)$. The WHSC1-S172A protein level, but not its mRNA level, was largely reduced compared with its WT counterparts (Figure 5I), whereas MG132 treatment attenuated WHSC1 protein degradation (Figure 5I and Supplemental Figure 5H). With the diminished phosphorylation of WHSC1 in S172A cells, the S172A 
mutant protein exhibited a profound increase in polyubiquitination (Figure 5J). In keeping with the loss-of-function phenotype associated with WHSC1, S172A cells showed pronounced defects in cell migration and anchorage-independent growth (Supplemental Figure 5I). More importantly, inoculations of control and S172A mutated cells into nude mice revealed that the inhibition of WHSC1-S172 phosphorylation greatly diminished metastatic lesions in the limbs, indicating that WHSC1 protein stabilization is essential for its function in promoting PCa metastasis (Figure $5 \mathrm{~K}$ ). The pathological association between AKT signaling and WHSC1 expression was further illustrated by patient specimen analysis. We performed IHC staining for phospho-AKT and WHSC1 on PCa TMAs (an Asian radical prostate cohort) and observed a positive correlation between WHSC1 and phospho-AKT levels (Figure 5L).

AKT phosphorylates WHSC1 to counteract CRL4 $4^{\text {Cdt2 }}$-dependent degradation. Next, we sought to determine the underlying mechanism by which AKT stabilizes the WHSC1 protein. It is known that AKT influences substrate protein ubiquitination and proteasome-dependent degradation through altering interactions between substrate proteins and their E3 ubiquitin ligases in a phosphorylation-dependent manner (43-45). Thus, we hypothesized that the AKT-mediated phosphorylation of WHSC1 altered the recruitment of E3 ligases and thereby affected its ubiquitination-mediated degradation. To this end, we explored tandem affinity purification of WHSC1 followed by mass spectrometric analysis to identify WHSC1-interacting proteins. Interestingly, two E3 ligase-related proteins, DNA damage binding protein 1 (DDB1) and CDT2 (also known as CDC10-dependent transcript 2, DTL, DACF2), were detected in WHSC1 immunoprecipitates (Figure 6A). The cullin 4 (CUL4) subfamily members CUL4A and CUL4B use DDB1 as an adaptor and DDB1 and CUL4-associated factors (DCAFs) as substrate receptors to recognize substrate proteins $(46,47)$. Thereafter, we examined the association between WHSC1 and CUL4 components by coimmunoprecipitation. Figure 6B indicates that CUL4B, DDB1, and CDT2, but not CUL4A, could be detected in WHSC1 immunoprecipitates. Consistently, silencing CUL4B and CDT2, but not CUL4A, elevated WHSC1 protein levels in 293T cells (Supplemental Figure 6A), and similar results were observed in PCa cells (Figure 6C).

To determine whether degradation of WHSC1 by CUL4B was regulated by AKT-mediated WHSC1-S172 phosphorylation, we performed an in vitro ubiquitination assay to determine the ability of CUL4B to add polyubiquitin chains to recombinant WT, S172A, and S172D WHSC1 proteins. To accomplish this, CUL4B ligase complexes were purified from Flag-CUL4B-overexpressing 293T cells, and WT and S172-mutated WHSC1 proteins were produced and purified from a baculovirus-insect cell expression system. As indicated in Figure 6D, IB with both anti-ubiquitin and anti-WHSC1 antibodies revealed that polyubiquitinated forms of WHSC1 were markedly enhanced when an alanine was substituted for WHSC1-S172, whereas phospho-mimic WHSC1-S172D protein exhibited significantly less polyubiquitination compared with WT protein. Similarly, when WHSC1-S172A protein was expressed in $293 \mathrm{~T}$ cells, it showed significantly more polyubiquitination compared with WT WHSC1 (Supplemental Figure 6B). As a negative control, the specificity of the ubiquitination assay was demonstrated by the enzymatic dead CUL4B (CUL4B-DN)
(Supplemental Figure 6B). Consistent with above observations, inactivation of CUL4B in an AKT-depleted or S172A-mutated cell line largely restored the WHSC1 protein level (Figure 6E), and cell migration was enhanced to an extent similar to that of WT cells (Figure 6F). These results indicate that CUL4B ${ }^{\mathrm{Cdt} 2}$-mediated degradation of WHSC1 is counteracted by the AKT-induced phosphorylation of WHSC1-S172.

Next, we aimed to elucidate the underlying mechanism by which phosphorylation of WHSC1-S172 prevents CUL4Bmediated destruction of WHSC1. Interestingly, when S172A and S172D were overexpressed in $293 \mathrm{~T}$ cells, we found that S172A enhanced while S172D diminished its interaction with CUL4B (Supplemental Figure 6C), suggesting that the phosphorylation of WHSC1-S172 prevented its association with CRL4 ${ }^{\mathrm{Cdt} 2} \mathrm{E} 3$ ligase. In line with this notion, inhibition of AKT by LY294002 treatment enhanced the interaction between endogenous WHSC1 and CUL4B, DDB1, and CDT2 proteins (Figure 6G). Similar results were obtained in WT and S172A-mutated PC3 cells. In agreement with the reduction in the phosphorylation and the increase in the polyubiquitination of WHSC1, we detected a more pronounced association of WHSC1 with CUL4B, DDB1, and CDT2 in S172A cells compared with WT cells (Figure 6H). These results highlight that AKT mediation of S172 phosphorylation abolishes the recognition of WHSC1 by CRL4 ${ }^{\text {Cdt2 }}$ E3 ligase.

The AKT/WHSC1/RICTOR cascade presents a positive-feedback loop within PCa cells to stimulate AKT signaling. To illustrate the mechanisms by which WHSC1 cooperated with PTEN loss to promote PCa progression, we conducted transcriptome analyses with or without WHSC1 depletion. As expected, Ingenuity Pathway Analysis (IPA) indicated that "molecular mechanisms of cancer" is one of the most significantly altered pathways (Supplemental Figure 7A). More importantly, Kyoto Encyclopedia of Genes and Genomes (KEGG) pathway analysis revealed that "regulation of actin cytoskeleton," "focal adhesion," and "mTOR signaling" were prominently enriched gene set concepts in the absence of WHSC1 (Figure 7A). As all of these processes greatly influence PCa progression and metastasis, we attempted to identify the genes directly regulated by WHSC1 to modulate these key processes.

Given that mTOR signaling might be altered in the absence of WHSC1, and we showed that AKT phosphorylated and stabilized WHSC1 in PCa cells, we first examined whether WHSC1 could enhance AKT activation and thereby lead to the vicious feedback stimulations of AKT signaling in PCa cells. In line with this hypothesis, we observed that depletion of WHSC1 profoundly inhibited AKT signaling activity, as reflected by the reduced phosphorylation of AKT (S473), FOXO1, S6, and mTOR (Supplemental Figure 7B). Conversely, overexpression of WHSC1, but not SET domain-truncated WHSC1, resulted in hyperactive AKT signaling (Supplemental Figure 7, B and C). Based on the results from cell culture studies, we further extended analyses to our mouse models. Immunostaining revealed there was a significant increase in phospho-AKT and phospho-S6 staining in tumor cells expressing a high level of WHSC1 (PTEN ${ }^{\mathrm{PC}-/-} \mathrm{WHSC1}^{\mathrm{PCOE} /+}$ ). Conversely, the staining intensities were substantially reduced in the tumor sections of $\mathrm{PTEN}^{\mathrm{PC}-/-} \mathrm{WHSC1}^{\mathrm{PC}-/-}$ mice (Figure 7B). Western blotting verified this observation, as indicated by enhanced or reduced phosphorylation of S6 and mTOR in prostate lysates derived from 
A

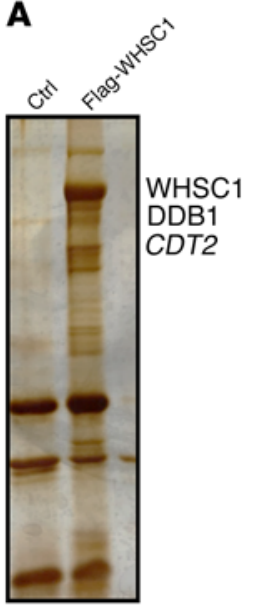

B

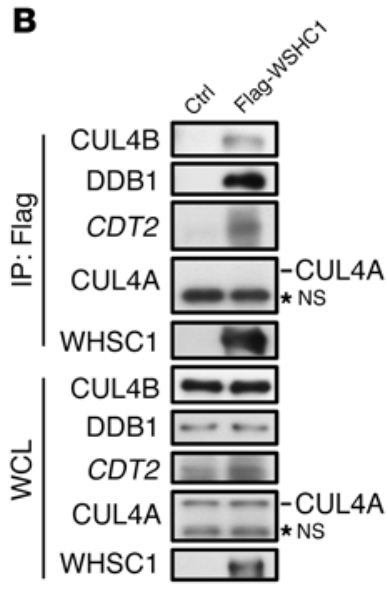

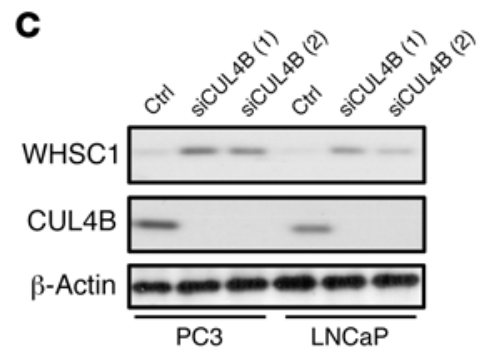

D WSCH1 $1(\mathrm{WT})_{+}+++-++$ WHSC1-S172A - - - - + WHSC1-S172D ----+CUL4B complex --+++++ E1 \& E2 \& ATP -+-++++

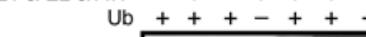

IB: WHSC

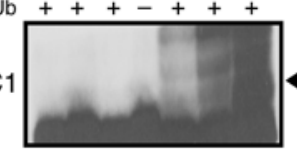
४Ub-WHSC 1

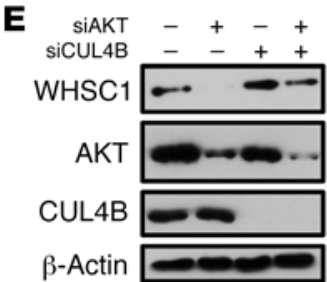

G

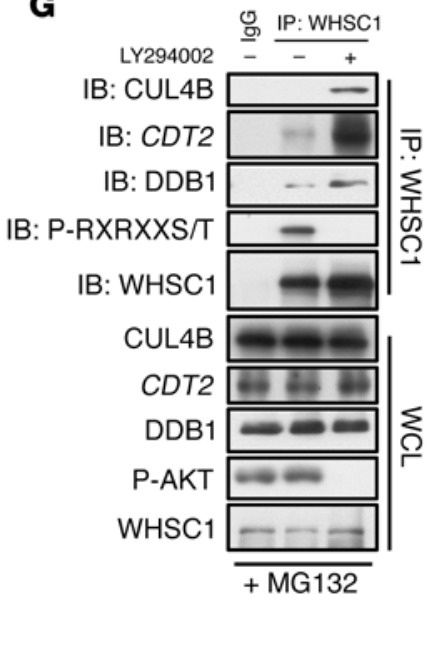

IB: Ub

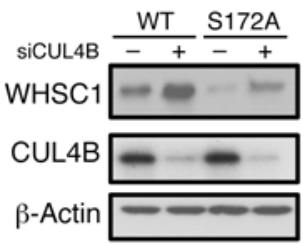

H

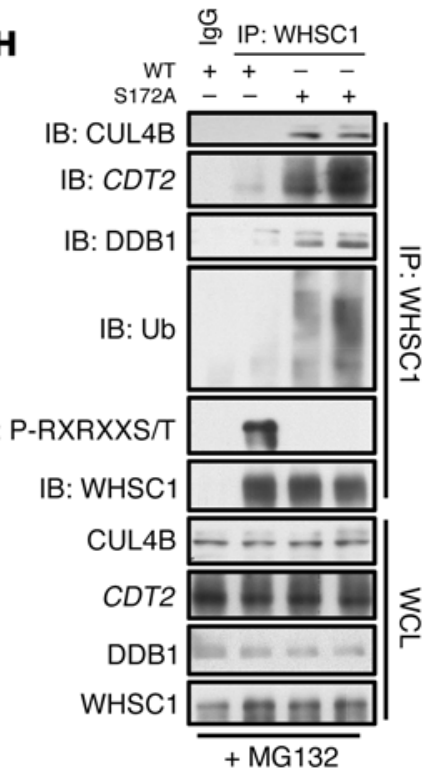

Figure 6. AKT phosphorylates WHSC1 to prevent CUL4B ${ }^{\text {Cdt2 }}$-dependent degradation. (A) A tandem affinity-purified Flag-WHSC1 protein complex from $293 T$ cells was resolved and visualized by silver staining. (B) IB analysis of the indicated protein in WCL and immunoprecipitates derived from 293T cells transfected with Flag-WHSC1. (C) IB analysis of WCL transfected with CUL4B siRNA in PC3 and LNCaP cells. (D) WT, WHSC1-S172A, and WHSC1-S172D proteins were incubated with and without CUL4B complex components, recombinant Ub, ATP, E1, and E2 enzymes as indicated and then subjected to Western blotting. (E) IB analysis of WCL in control and CUL4B knockdown cells with or without AKT depletion (left), or in WT and S172A mutated PC3 cells with or without CUL4B knockdown (right). (F) Migration abilities of the parental and S172A mutant cells with or without CUL4B depletion. Data are presented as the mean \pm SEM. Two-way ANOVA followed by Tukey's multiple comparisons test, ${ }^{* *} P<0.01(n=3)$. (C) IB analyses of WCL and anti-WHSC1 immuno-

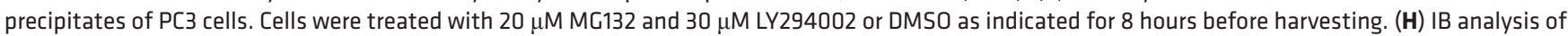
WCL from WT and S172A PC3 cells and anti-WHSC1 immunoprecipitates as indicated.

$\mathrm{PTEN}^{\mathrm{PC}-/-}$ WHSC1 ${ }^{\mathrm{PCOE} /+}$ or PTEN ${ }^{\mathrm{PC}-/-} \mathrm{WHSC1}^{\mathrm{PC}-/-}$ mice (Figure $7 \mathrm{C}$ ). Thus, our results highlight the notion that WHSC1 engendered the acquisition of hyperactive AKT signaling in prostate tumors.

Interestingly, gene expression profiling revealed reduced RICTOR expression in WHSC1-depleted cells. Since RICTOR, a key component of the mTORC2 complex, can phosphorylate AKT at S473 to induce feedback activation of AKT signaling, we postulated that WHSC1 modulated AKT activity via regulation of RICTOR expression. Indeed, WHSC1 positively regulated RICTOR expression in PCa cells and GEM models (Figure
7, C-E). To determine whether WHSC1 enhances AKT signaling through direct regulation of RICTOR expression, we conducted ChIP-Seq (chromatin immunoprecipitation followed by high-throughput sequencing) to characterize the differences in the genomic distribution of H3K36me 2 codes between WT and WHSC1-deficient PC3 cells. In agreement with previous findings, the H3K36me2 signal was preferentially enriched in intragenic regions on a genome-wide scale in WT cells (Supplemental Figure 7D). Comparison of ChIP-Seq profiles in control and WHSC1-depleted cells revealed a total of 2,171 genes displaying 
A
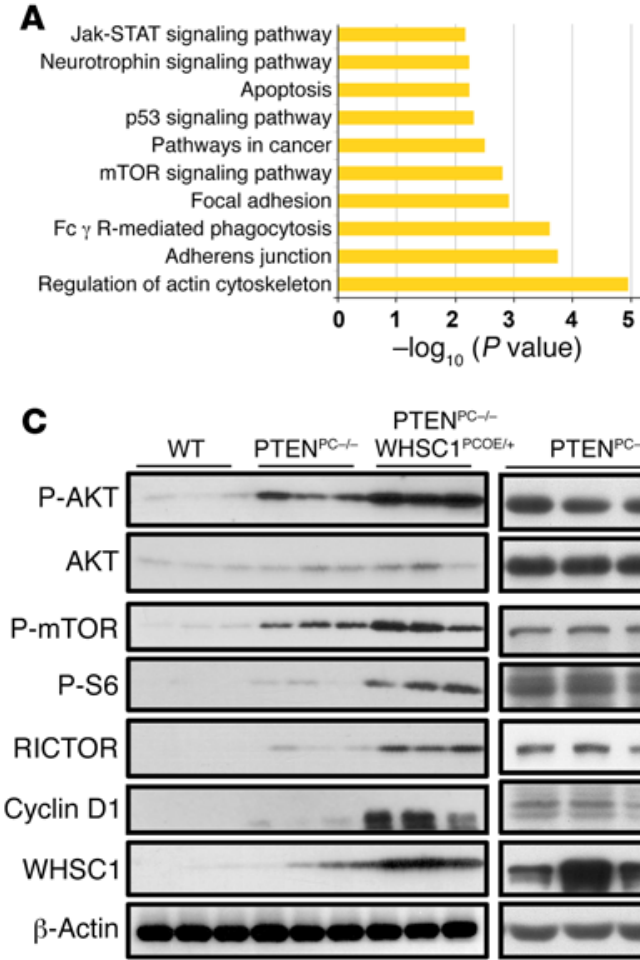

$\mathbf{F}$
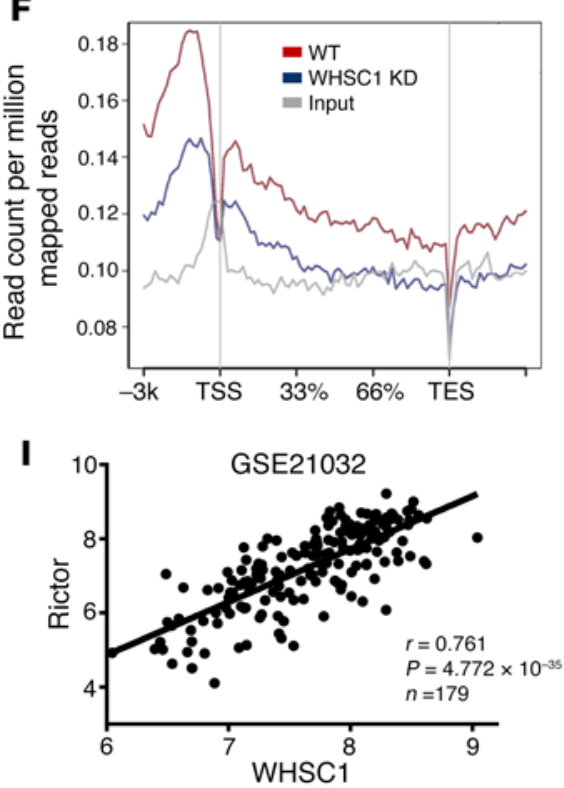

J

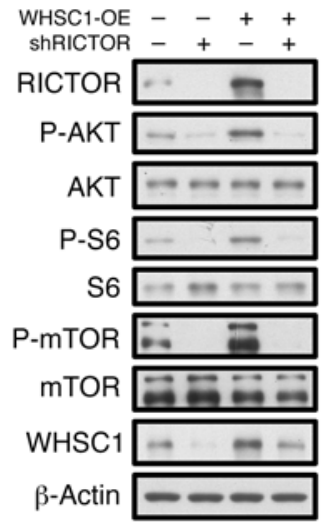

B

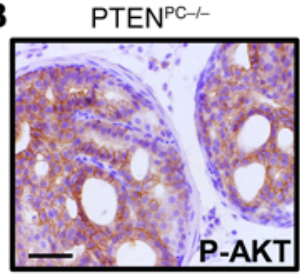

$\mathrm{PTEN}^{\mathrm{PC}-1-} \mathrm{WHSC1}^{\mathrm{PC}-1-} \mathrm{PTEN}^{\mathrm{PC}-1-} \mathrm{WHSC}^{\mathrm{PCOE} /+}$
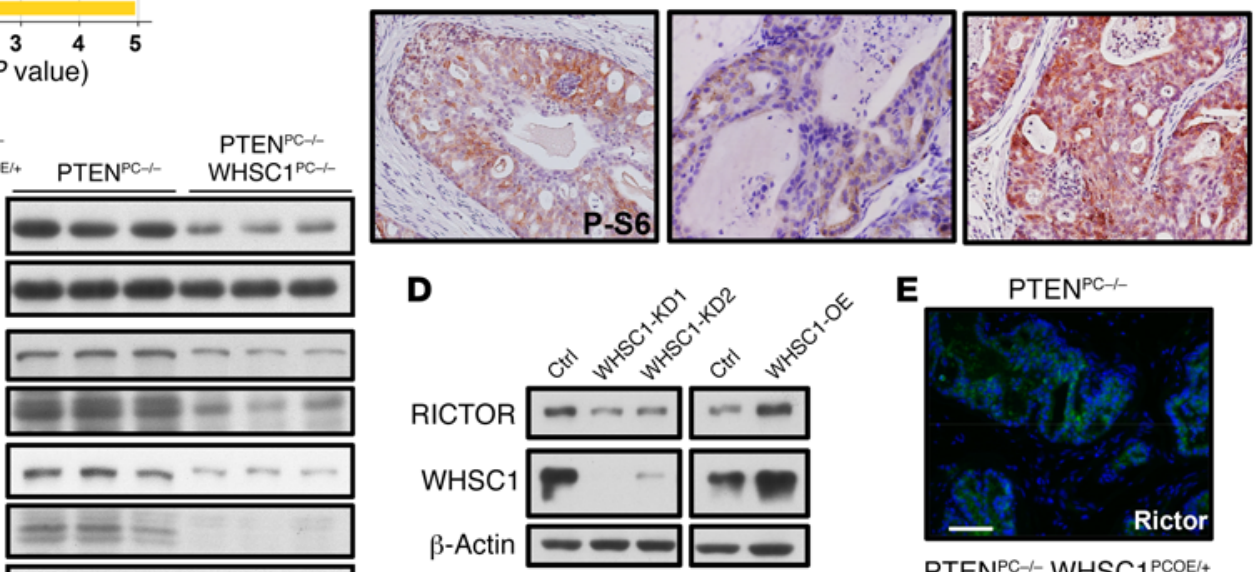

E $\quad \mathrm{PTEN}^{\mathrm{PC}-1-}$
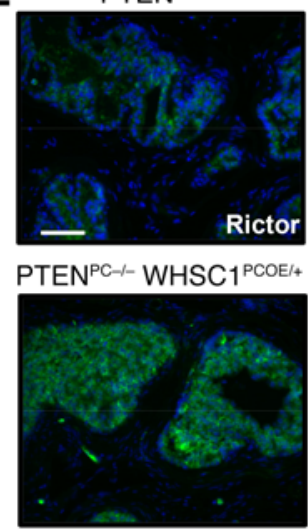

WHSC1 KD versus WT

H

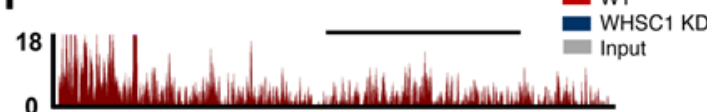

H3K36me2 Expression levels modifications reduce decrease

$$
18
$$

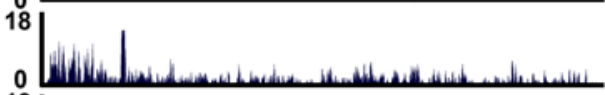

${ }_{0}^{18}$

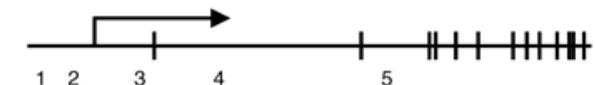

WHSC1 ChIP

H3K36me2 ChIP
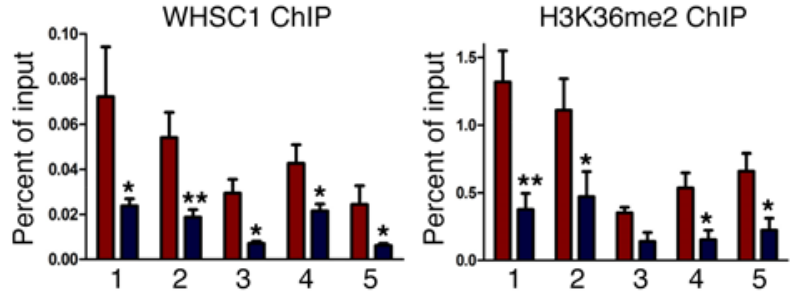
Figure 7. WHSC1 stimulates AKT signaling through direct regulation of RICTOR expression. (A) KEGG analysis shows the altered pathways in the absence of WHSC1. (B) IHC staining of phospho-AKT (S473) (top) and phospho-S6 (bottom) levels in the prostate sections of PTEN ${ }^{\mathrm{PC}-1-}$, PTEN ${ }^{\mathrm{PC}-1-}$ $\mathrm{WHSC}^{\mathrm{PC}-1-}$, and $\mathrm{PTEN} \mathrm{PC}-1-^{\mathrm{PC}} \mathrm{WHSC} 1^{\mathrm{PCOE} /+}$ mice. (C) Western blot analysis of WCL of AP from 4-month-old mice as indicated. Blot images are derived from replicate samples run on parallel gels. (D) IB analysis of RICTOR expression in WHSC1-KD and WHSC1-OE PC3 cells. (E) IHC of RICTOR expression in $\mathrm{PTEN}^{\mathrm{PC}-{ }_{-}-}$and $\mathrm{PTEN} \mathrm{PC}-1-^{\mathrm{P}} \mathrm{WHSC}^{\mathrm{PCOE} /+}$ mice. $(\mathbf{F})$ Tag density profile of H3K36me2 distribution of genes showing the decrease in H3K36me2 modifications in WHSC1-silenced cells. (G) Venn diagram indicating the overlap of genes with reduced expression (gray) and H3K36me2 modifications (yellow) in WHSC1 knockdown cells. (H) Snapshot of H3K36me2 ChIP-Seq signals at the Rictor gene locus in control and WHSC1 knockdown cells (top). ChIP-quantitative PCR WHSC1 and H3K36me2 signals in control and WHSC1-depleted cells using the indicated primer pairs (bottom). The location of the ChIP primer pairs used in the present study is denoted as numbers. Error bars indicate the SEM from 3 experiments. Student's $t$ test, ${ }^{*} P<0.05,{ }^{*} P<0.01$. (I) The association (by Pearson's) between WHSC1 and RICTOR transcripts in patients (GSE21032, $n=179$ ). (J) IB analysis of WCL (left) and anchorage-independent growth (right) in control and WHSC1-overexpressing PC3 cells with or without RICTOR depletion. Scale bars: $50 \mu \mathrm{m}$ (B and $\mathbf{E}), 50 \mathrm{~kb}(\mathbf{H})$.

reduced H3K36me2 levels after WHSC1 silencing (Supplemental Figure 7E). In line with the notion that $\mathrm{H} 3 \mathrm{~K} 36 \mathrm{me} 2$ modification is critical for gene transcription and elongation, the difference in the peaks between control and knockdown cells was more obvious within the promoter areas and throughout gene bodies (Figure 7F). To further correlate chromatin bindings with direct gene regulation, we integrated the WHSC1-dependent transcriptome and noticed that a total of 153 genes might be directly regulated by WHSC1, as they showed a reduction in both $\mathrm{H} 3 \mathrm{~K} 36 \mathrm{me} 2$ modifications and expression levels in the absence of WHSC1 (Figure $7 G)$. More importantly, KEGG pathway analysis of the overlapping genes revealed enrichment of gene set concepts such as "focal adhesion," "regulation of actin cytoskeleton," and "pathways in cancer" (Supplemental Figure 7G), emphasizing the role of WHSC1 in tumorigenesis.

Focusing on the regulation of RICTOR expression, we observed a significant reduction in $\mathrm{H} 3 \mathrm{~K} 36 \mathrm{me} 2$ signals in the Rictor gene locus, especially within the $5^{\prime}$-UTR and promoter regions in WHSC1-depleted PC3 cells (Figure 7H, top). Next, a systematic, quantitative direct ChIP strategy was conducted to determine whether WHSC1 indeed directly regulated RICTOR expression. ChIP-quantitative PCR examination confirmed that WHSC1 depletion decreased $\mathrm{H} 3 \mathrm{~K} 36 \mathrm{me} 2$ signals and WHSC1 recruitment to the Rictor gene locus (Figure 7H, bottom). Likewise, the direct regulation of RICTOR expression by WHSC1 was strengthened in patients; RICTOR expression positively correlated with WHSC1 transcripts in patient specimens (using data set GSE21032; Figure 7I). To clarify the functional importance of the WHSC1/RICTOR axis, we depleted RICTOR expression in WHSC1-overexpressing cells and found that RICTOR depletion diminished AKT phosphorylation and anchorage-independent growth in WHSC1-overexpressing cells to an extent similar to that of control cells (Figure 7J). Notably, in accordance with the previous statement that AKT stabilized the WHSC1 protein, RICTOR depletion also reduced WHSC1 levels (Figure 7J, left). Collectively, our results highlight that the AKT/WHSC1/RICTOR cascade represents a positive-feedback loop within PCa cells to viciously activate AKT signaling and thereby promote PCa malignancy.

WHSC1/Rac1 signaling potentiates the metastatic traits of $P C a$ cells. Our results demonstrated that WHSC1 plays an integral role in abnormal PI3K/AKT signaling to promote prostate tumorigenesis. However, in addition to AKT/mTOR signaling, unbiased RNA-Seq and ChIP-Seq analyses revealed that focal adhesion and the actin cytoskeleton, which are also important for cell motility and tumor metastasis, might also be altered in the absence of WHSC1 (Figure 7A and Supplemental Figure 7G). We proposed that WHSC1 also modulated these key processes to promote PCa metastasis. To verify this notion, we first examined stress fibers and focal adhesion in WHSC1-deficient cells by staining for phalloidin, P34-Arc (a subunit of ARP2/3), phospho-FAK, and phospho-paxillin. Indeed, WHSC1 deficiency led to defects in cell spreading and protrusions without a clear leading edge of lamellipodium (Figure 8A). In addition, WHSC1-depeleted cells exhibited adhesion foci defects at the cell periphery, as indicated by phospho-FAK and phosphopaxillin staining (Figure 8A and Supplemental Figure 8A). Conversely, WHSC1 overexpression improved cellular spreading and polarity in comparison with control cells (Figure 8A). Taken together, our data indicate that WHSC1 is an upstream regulator of cytoskeletal remodeling and focal adhesion, thus providing a plausible explanation for why WHSC1 promotes metastasis.

Given that Rho signals are critical for cytoskeletal remodeling and tumor metastasis (48) and that our IPA analysis suggested that "regulation of actin-based motility by Rho" is sensitive to WHSC1 depletion (Supplemental Figure 7A), we referenced gene expression profiles to characterize whether WHSC1 modulated Rho expression in PCa cells. Although examination of RhoA, RhoB, and $\mathrm{RhoC}$ levels indicated that there were no appreciable alterations after WHSC1 dysregulation, we found that Rac1 expression was prominently altered in WHSC1-depleted or WHSC1-overexpressing cells (Figure 8B). Consistently, glutathione $S$-transferase pull-down analysis indicated that active Rac1, Rac1-GTP, was simultaneously reduced with the downregulation of WHSC1 expression (Supplemental Figure 8B). In support of this biochemical characterization, gene set enrichment analysis, a computational method that detects coordinated changes in the expression of groups of functionally related genes, revealed a large fraction of Rac1 downstream genes that displayed significant alterations in WHSC1-depleted cells (Figure 8C), indicating that Rac1 signaling on a genomic basis was compromised in the absence of WHSC1. Next, we extended our analysis to a GEM model in which WHSC1 overexpression potentiated indolent PTEN-null tumors developing into metastatic PCa. Indeed, IHC analysis showed that Rac1 expression was elevated in the tumors of $\mathrm{PTEN}^{\mathrm{PC}-/-} \mathrm{WHSC1}^{\mathrm{PCOE} /+}$ mice, especially within invasive foci (Figure 8D). This observation was further reinforced by Western blot analysis. There were significant increases in the total Rac1 and phospho-PAK1 levels when tumor cells harbored higher WHSC1 expression levels (Figure 8E).

Having demonstrated that WHSC1 modulated Rac1 expression, we conducted rescue experiments and found that the depletion of Rac1 expression in WHSC1-overexpressing cells attenuated the acceleration of cellular migration caused by WHSC1 overexpression (Supplemental Figure 8, C and D). Similar results were obtained by staining of stress fibers, lamellipodia, and focal 
A Ctrl
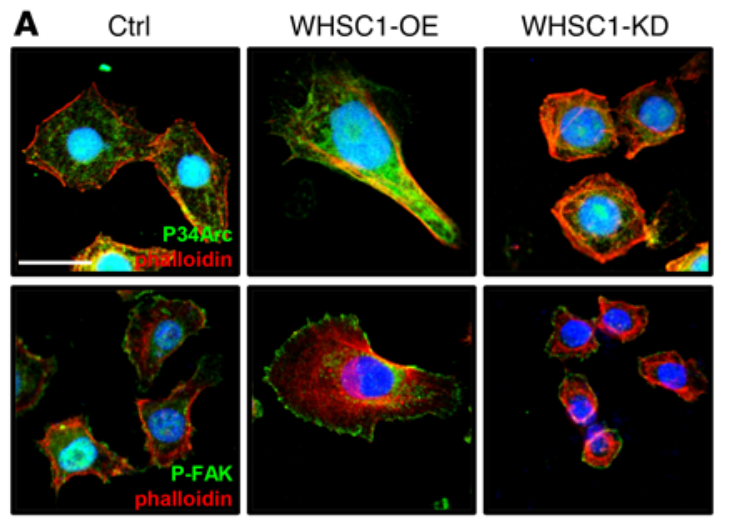

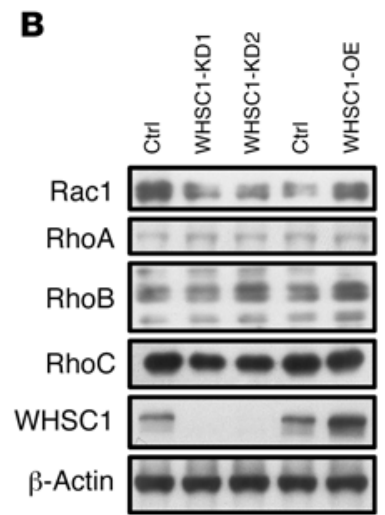

C

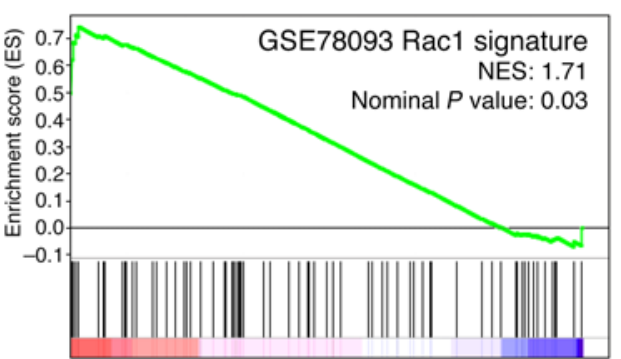

WHSC1 WT vs. WHSC1-KD
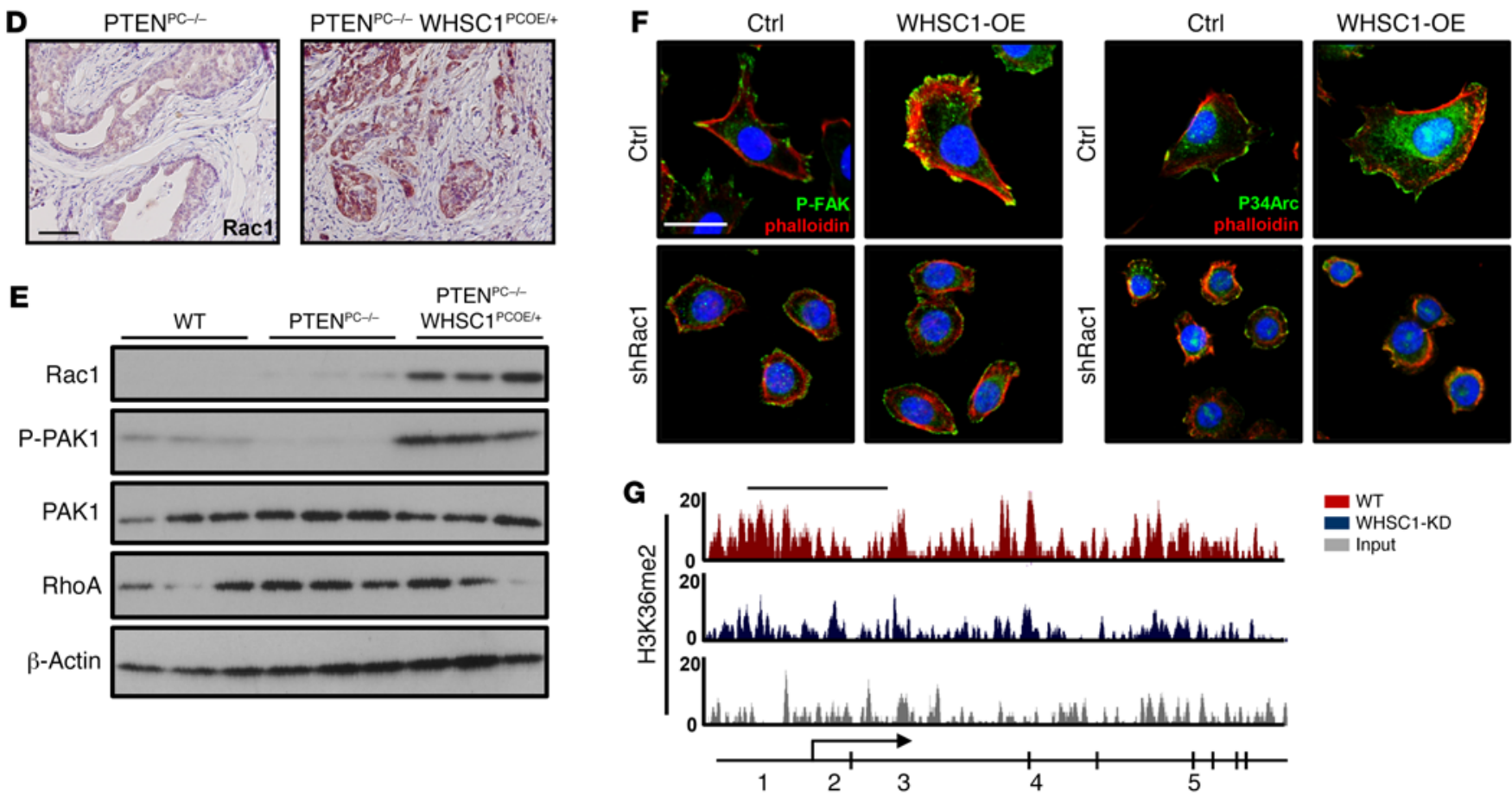

Figure 8. WHSC1 modulates the Rac1 signal to promote PCa metastasis. (A) Immunostaining as indicated in control, WHSC1-OE, and WHSC1-KD PC3 cells. (B) IB analysis of WCL from control, WHSC1-KD, and WHSC1-OE PC3 cells. (C) Gene set enrichment plots of differentially expressed genes belonging to the Rac1 pathway associated with WHSC1 downregulation. $P$ value is determined by GSEA software. (D) IHC staining of Rac1 expression in DLP sections from 4-month-old PTEN ${ }^{\mathrm{PC}-I_{-}}$and PTEN ${ }^{\mathrm{PC}-1-}$ WHSC1 ${ }^{\mathrm{PCOE} /+}$ mice. (E) Western blot analysis of the indicated protein in WCL of AP from 4-month-old WT, $\mathrm{PTEN}^{\mathrm{PC}-1 \text {, }}$, and PTEN $\mathrm{PC}-1-^{\mathrm{W}}$ WHSC1 ${ }^{\mathrm{PCOE} /+}$ mice. (F) Immunostaining as indicated in control and WHSC1-OE PC3 cells with or without Rac1 depletion. (C) Snapshot of H3K36me2 ChIP-Seq signals at the RAC1 gene locus in control and WHSC1-silenced cells. Scale bars: $10 \mu \mathrm{m}(\mathbf{A}$ and F), $50 \mu \mathrm{m}$ (D), $10 \mathrm{~kb}(\mathbf{G})$,

adhesions, which revealed that cytoskeletal remodeling and stress fiber reorganization were reversed in the rescued cells (Figure 8F). In line with this observation, overexpression of constitutively active Rac1 (Rac1-Q61L), but not dominant-negative Rac1-T17N, neutralized the cell migration defects elicited by WHSC1 depletion (Supplemental Figure 8, E and F). Thus, the effect of WHSC1 on cytoskeletal remodeling and cell migration is mediated via regulation of Rac1 expression.

Focusing on the regulation of Rac1 expression, ChIP-Seq results revealed a global reduction in H3K36me2 modifications spanning from the $5^{\prime}$-UTR area to the body of the RAC1 gene (Figure 8G). Consistent with reduced WHSC1 recruitment to the RAC1 gene locus, ChIP-quantitative PCR analyses indicated that the depletion of WHSC1 reduced $\mathrm{H} 3 \mathrm{~K} 36 \mathrm{me} 2$ modifications across the various sites examined (Figure 9A). In addition, a large set of areas displayed an obvious decrease in $\mathrm{H} 3 \mathrm{~K} 9 \mathrm{ac}$ modifications accompanied by a concomitant increase in H3K27me3 levels (Figure 9A), suggesting that the loss of WHSC1 led to more condensed chromatin in the RAC1 gene locus and thereby blunted its transcription in PCa cells. Finally, the regulation of the WHSC1/Rac1 axis was strengthened by analysis of the public data sets. Similar to increased WHSC1 expression in PCa patients, the signatures of WHSC1 and Rac1, as defined by expression profile changes, were both significantly elevated in patients with metastatic PCa (Supplemental Figure 8G), emphasizing the potential importance of WHSC1 and Rac1 signaling in PCa metastasis. In addition, a positive and significant correlation between WHSC1 and Rac1 was identified in prostate tumors (GSE21032; Supplemental Figure 

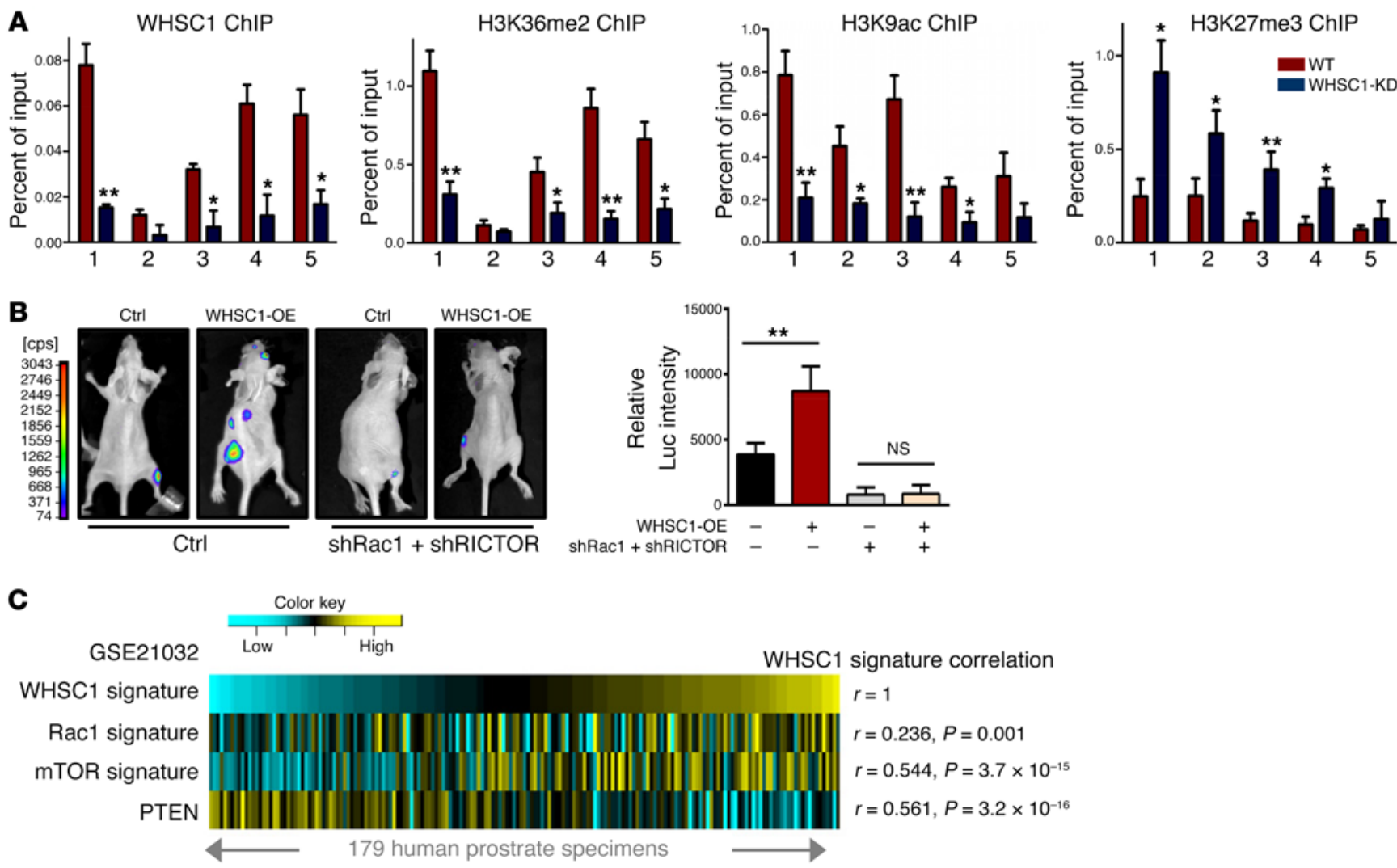

Figure 9. WHSC1 modulates the Rac1 signal to promote PCa metastasis. (A) ChIP-quantitative PCR signals of WHSC1, H3K36me2, H3K27me3, and H3K9ac in control and WHSC1-depleted cells using the indicated primer pairs. Student's $t$ test, ${ }^{*} P<0.05,{ }^{* *} P<0.01$. (B) Representative bioluminescence results show tumor metastasis in control and WHSC1-OE PC3 cells with or without Rac1 and RICTOR knockdown (left). BLI quantitation of bioluminescent signals, which are calculated as the means \pm SEM, is shown in the right panel ( $n=8$ per group). Two-way ANOVA followed by Tukey's multiple comparisons test, ${ }^{* *} P<0.01$. (C) Correlations (by Pearson's) between the WHSC1 signature (GSE84868), Rac1 signature (GSE78093), mTOR signature (GSE1413), and PTEN expression within normal and PCa specimens (CSE21032, $n=179$ ) are shown. Yellow, high-signature scoring in prostate tumor specimens; blue, low-signature scoring.

$8 \mathrm{H})$. Together, these findings demonstrate that WHSC1 serves as an H3K36 dimethyl transferase that directly regulates Rac1 expression and thereby confers metastatic traits on PCa cells.

Finally, to verify whether the mechanism identified here functionally contributes to the metastatic phenotype in mouse models, we simultaneously depleted RICTOR and Rac1 expression in WHSC1-overexpressing cells (Supplemental Figure 8I) and performed an intracardiac injection assay to characterize whether this depletion could eliminate the effect of WHSC1 on PCa metastasis. As shown by BLI, depletion of Rac1 and RICTOR inhibited the metastasis of tumor cells to the bone, as expected (Figure 9B). More importantly, we detected that reduced expression of Rac1 and RICTOR in WHSC1-overexpressing cells abrogated the invasion of tumor cells within the skeleton (Figure 9B). This observation was further strengthened by $\mathrm{x}$-ray imaging, which showed that depletion of Rac1 and RICTOR expression diminished tumor invasion within the skeleton in WHSC1-OE tumors to an extent similar to that of the control cells (Supplemental Figure 8J). The functional cooperation between WHSC1 and key intracellular molecules (PTEN or AKT/RICTOR/Rac1) was illustrated by transcriptomic signature analysis in patients. There was a significant and positive correlation among WHSC1 activity, mTOR and Rac1 signaling activation, and PTEN reduction in PCa patients (using data set GSE21032; Figure 9C). Thus, our GEM model, togeth- er with an ex vivo PCa metastatic model and functional characterization, collectively demonstrates that WHSC1 cooperates with PTEN loss to aggravate PCa progression via stimulation of mTORC2/AKT and Rac1 signaling.

Prognostic potential of WHSC1 and PTEN signaling in human $P C a$. We next aimed to assess whether genetic cooperation between WHSC1 signaling and PTEN loss in mice could be recapitulated in human PCa. We first examined how robustly WHSC1, PTEN, mTOR, and Rac1 signaling activity stratifies the biochemical recurrence (BCR) in patients (using the data set of Taylor et al., GSE21032; ref. 4). Though mTOR activity was not significantly associated with BCR, univariate analysis indicated that the PTEN, WHSC1, and Rac1 signatures independently provided predictive power to stratify patients into high- and low-risk groups (Supplemental Table 3).

Given that current diagnostic methods, including prostatespecific antigen (PSA) testing and the Gleason score, inaccurately distinguish indolent tumors versus aggressive $\mathrm{PCa}$, we next posited that WHSC1 and PTEN signaling may carry prognostic value to stratify the metastatic risk in human PCa. To this end, we performed multivariate analysis to determine whether WHSC1 and PTEN could improve the diagnostic accuracy of PSA and the Gleason score in stratifying the risk of BCR in patients (using data set GSE21032). According to log-rank tests, multivariate Cox proportional hazards regression analysis revealed that WHSC1 
Table 1. Multivariate Cox regression analysis in GSE21032 $(n=140)$

$\begin{array}{lccc}\text { Multivariate Cox } & \boldsymbol{P} \text { value } & \text { HR } & \text { 95\% Cl } \\ \text { Cleason score } & 0.002 & 2.26 & 1.36-3.74 \\ \text { PSA } & 0.028 & 1.77 & 1.07-2.95 \\ \text { WHSC1 } & 0.024 & 1.67 & 1.07-2.59 \\ \text { PTEN } & 0.05 & 0.65 & 0.42-1.01 \\ \text { Rac1 signature } & 0.146 & \text { NA } & \text { NA }\end{array}$

and PTEN both carried an independent predictive power to further enhance the prognostic accuracy of the PSA level and Gleason score (Table 1). As indicated by Kaplan-Meier plot, combined levels of PTEN and WHSC1, together with the Gleason score or PSA, stratified the patient cohort into 2 groups ( $K$-means clustering) that displayed significant differences in the risk of BCR (HR $=3.9, P<0.0001$; HR $=2.2, P=0.027$; Figure 10A). By $C$-statistics analyses, we showed that WHSC1 and PTEN together (2-gene model) carried independent prognostic information, as it can enhance the prognostic accuracy of the Gleason score and PSA levels from $C$-index values ranging from 0.69 to 0.72 and 0.59 to 0.69 , respectively (Table 2). Encouraged by the revealed prognostic value, we repeated these results in our TMA by IHC staining with phospho-AKT and WHSC1 and observed similar results; the expression of WHSC1 together with higher phospho-AKT levels was also capable of enhancing the prognostic accuracy of the Gleason score and PSA in predicting BCR outcome (Supplemental Figure 9, A and B, and Supplemental Tables 3 and 4). Together, these results corroborate the biological significance of genetic cooperation between the signals of WHSC1 and PTEN loss in PCa metastasis, with potential importance for diagnosis.

\section{Discussion}

Activation of the PI3K/AKT signaling pathway by either PTEN loss or direct activation mutations is frequently observed in metastatic prostate tumors $(4,5)$. However, this alteration is insufficient to endow cells with metastatic traits. Employing GEM models and patient specimen analysis, we found that WHSC1 represents an "epigenetic hit," collaborating with PTEN loss to promote metastatic transformation. Though we showed that WHSC1 overexpression in PTEN-null mice greatly enhances tumor cell metastasis to the lymph nodes and lungs, bone metastatic lesions were not detected in overexpression mice. Currently, there are no GEMs that reliably display bone metastasis in vivo, which represents a major limitation in the study of advanced PCa. Thus, whether WHSC1 specifically plays critical roles in bone metastasis remains to be defined.

We demonstrated that WHSC1 expression is upregulated in the prostate epithelium in an AKT-dependent manner and is indispensably required for the malignancy of PTEN-null tumors. Mechanistically, we found that AKT phosphorylates WHSC1 to antagonize CRL4 ${ }^{\text {Cdt2 }}$-dependent degradation. Increased WHSC1 directly stimulates RICTOR expression, forming a positive-feedback loop to further enhance the activation of AKT signaling and WHSC1 expression (Figure 10B). Our findings are of high clinical rele- vance to metastatic $\mathrm{PCa}$, as up to $70 \%$ of metastatic tumors harbored PTEN loss or PI3K/AKT signaling hyperactivity mutations. The importance of regulating WHSC1 protein stability is substantiated by a recent study showing that the protein level of WHSC1 is dynamically regulated during cell cycle progression and promotes normal DNA replication (49). Interestingly, previous studies also indicated that EZH2 transcriptionally stimulates WHSC1 expression through negative regulation of microRNA expression (24), which partially explains why WHSC1 mRNA is also increased in metastatic tumors. Since multiple oncogenic insults, such as EZH2 and AKT signaling, cause an increase in WHSC1 in metastatic PCa, it is not surprising that a further increase in WHSC1 in $\mathrm{PTEN}^{\mathrm{PC}-/-}$ mice promotes indolent PTEN-null tumor cells to acquire a metastatic potential (Figure 10B). Collectively, these results highlight the complexity of signal coordination to enhance WHSC1 in PCa cells. Thus, fine-tuning of WHSC1 activity levels likely ensures a rapid and strictly bimodal response to diverse oncogenic stimulates and thereby epigenetically reprograms the tumor cells to acquire metastatic traits.

Deregulation of the epigenetic machinery is one of the main drivers of oncogenic transformation and cancer development. Emerging evidence indicates that deregulation of a single component of the machinery affects the global epigenetic landscape, including mutations in EZH2, and tet methylcytosine dioxygenase 2 (TET2), among others (50-52). Our results highlight that tumors bearing a high level of WHSC1 confer the metastatic traits of PCa cells through epigenetically reprograming the genome landscapes for a subset of genes. This notion was exemplified by examination of the RAC1 gene in the present study. We detected that WHSC1 depletion significantly increased H3K27me3 modifications concomitant with a reduction in histone codes for $\mathrm{H} 3 \mathrm{~K} 36 \mathrm{me} 2$ and $\mathrm{H} 3 \mathrm{~K} 9 \mathrm{ac}$ in the RAC1 gene locus, likely leading to more condensed chromatin to reduce Rac1 transcription. Intriguingly, ChIP-Seq analysis indicated that approximately $30 \%$ of genes on a genomic basis displayed significant reduction in $\mathrm{H} 3 \mathrm{~K} 36 \mathrm{me} 2$ codes in WHSC1-silencing cells compared with control cells. However, incorporation of the results from gene expression profiling in the same cells revealed that the changes in H3K36me2 occupancies were not tightly correlated with gene expression alterations, reflecting the complex coordination of various histone modifications under different insults. In addition, since the core subunits and associated factors of the WHSC1 complex are not elucidated, the underlying mechanisms

Table 2. C-statistics analysis reveals that a 2-gene set (WHSC1 and PTEN) improves the prognostic accuracy of the Gleason score and PSA

$\begin{array}{lcc}\text { Model } & \text { C-index } & 95 \% \text { Cl } \\ \text { Model 1. Cleason score } & 0.69 & 0.61-0.78 \\ \text { Model 2. PSA } & 0.59 & 0.52-0.65 \\ \text { Model 3. Two-gene set (WHSC1, PTEN) } & 0.63 & 0.56-0.71 \\ \text { Model 4. Cleason score + 2-gene set } & 0.72 & 0.63-0.82 \\ \text { Model 5. PSA + 2-gene set } & 0.69 & 0.60-0.78\end{array}$

CSE21032 (BCR cases, $n=36$; BCR-free cases, $n=104$ ). 
A
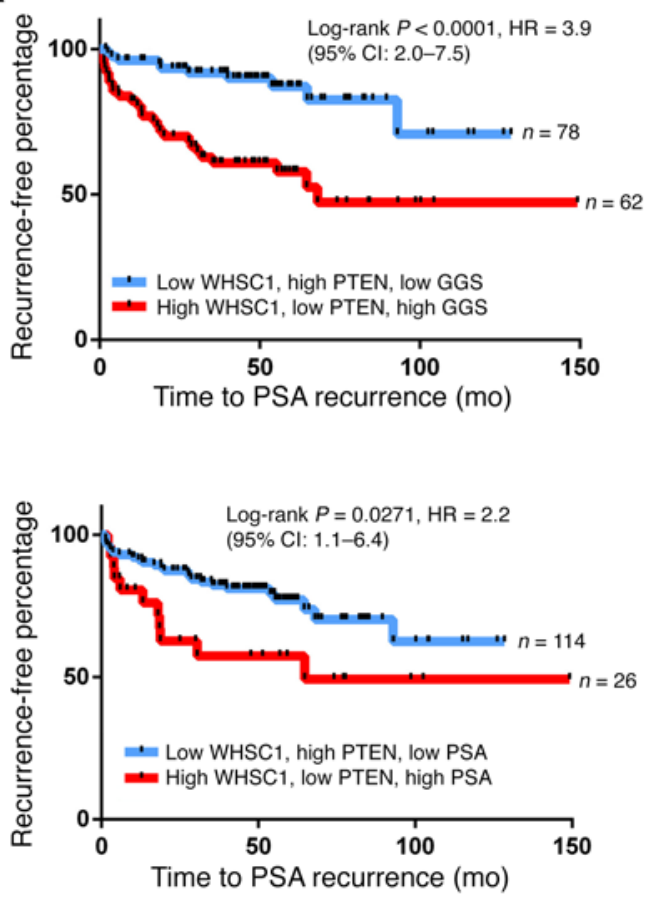

B

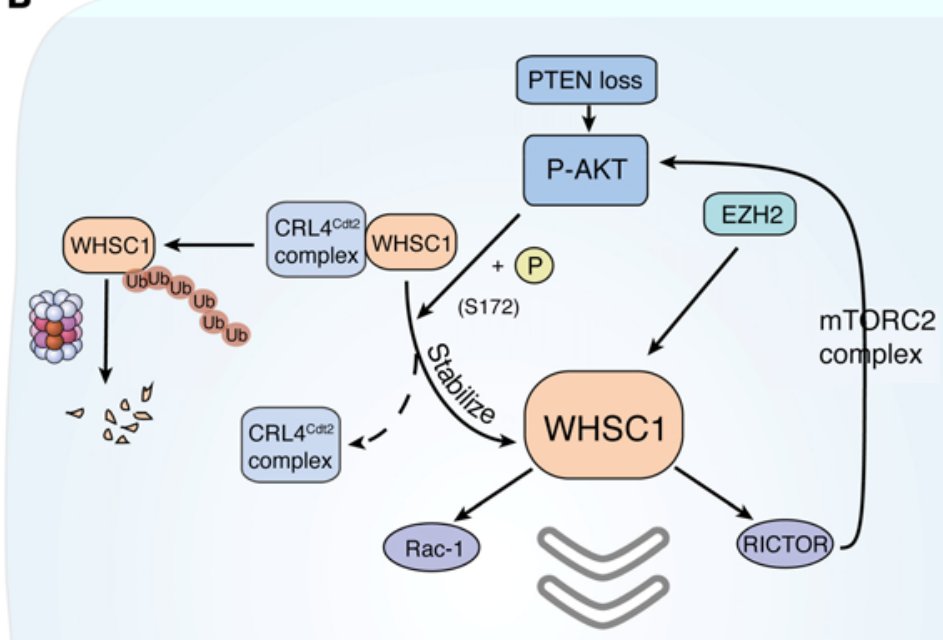

PCa progression and metastasis

is H3K36me2

WHSC1 low

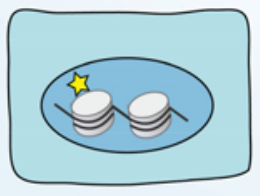

Indolent prostate tumor

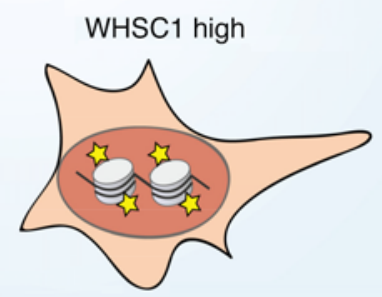

Metastatic PCa

Figure 10. Prognostic potential of WHSC1 and PTEN signaling in human PCa. (A) Kaplan-Meier plot ( $P$ values by log-rank test) of patients grouped using the combination of WHSC1, PTEN, and the Gleason score or PSA ( $K$-means clustering). (B) Model of WHSC1 in prostate tumorigenesis. PTEN inactivation to activate AKT directly phosphorylates WHSC1 and abolishes the recognition and degradation of WHSC1 by CRL4 ${ }^{\text {cdt2 }}$ E3 ligase. Increased WHSC1 in PCa cells transcriptionally upregulates the expression of RICTOR, forming a positive-feedback loop to further enhance the activation of AKT signaling and WHSC1 expression. WHSC1 also enhances the expression of Rac1, which contributes to the transformation of indolent prostate tumors to metastatic cancer. Thus, WHSC1 serves as an epigenetic integrator in mediating AKT, RICTOR, and Rac1 signaling crosstalk to drive PCa metastasis.

by which WHSC1 recognizes a specific set of genes remain undefined. Interestingly, motif analysis of WHSC1 intervals based on ChIP-Seq data revealed significant enrichment of transcription factors important for prostate tumorigenesis, such as NKX2.1, NKX3.1, and FOXO1 (Supplemental Figure 7F). Whether WHSC1 directly interacts with these transcription factors to promote PCa progression remains to be determined.

As WHSC1 is commonly dysregulated in human cancers and its function is largely dependent on enzymatic activity, inhibition of WHSC1 activity may have therapeutic implications for a diverse set of tumors. For PCa patient management, current methods of tumor stratification are based on clinicopathological factors, including the Gleason score, PSA, and tumor stages (2). However, these parameters are widely considered inaccurate for predicting patient outcome. Our results indicate that WHSC1, together with PTEN signaling, enhances the prognostic accuracy of PSA or the Gleason score for the prediction of BCR in patients. Though the TMA used in this study includes limited specimens, the results were consistent with public data set analysis. Thus, the present study provides insight to facilitate the development of a molecu- lar prognostic assay that may complement the current standard to improve evidence-based management of PCa patients. In summary, our findings highlight the role of WHSC1 in driving full malignant transformation of PTEN-null prostate tumors and have potential clinical relevance.

\section{Methods}

For expression plasmid, siRNA, cell culture, primer, colony formation assay, migration, ubiquitination, mass spectrometry, and in vitro kinase assay procedures, see the Supplemental Methods.

Animal experiments. All experiments were maintained in a specific pathogen-free facility. WHSC1 overexpression mice (WHSC $1^{\mathrm{OE} /+}$ ) were generated by our group using a previously described approach (11). Whsc $1^{7 / l}$ mice were generated by Beijing Biocytogen Co. Ltd., using conventional homologous recombination in embryonic stem cells, and the targeting strategy is indicated in Supplemental Figure 3A. Pten ${ }^{\pi / f}$ mice were obtained from Hong Wu at UCLA (8). The $P B^{C r e / t}$ transgenic mice were obtained from Fen Wang at the Institute of Bioscience and Technology, Texas A\&M Health Science Center (53). All the mice were backcrossed with C57BL/6 mice for at least 7 gen- 
erations. For intracardiac injection, nude mice aged 4-8 weeks were injected with $10^{6}$ PC3 cells. BLI was acquired with a NightOWL II LB 983 Imaging System (Berthold). Bone damage was detected by x-ray radiography with a Faxitron instrument (Faxitron Bioptics) and quantified using Image $(\mathrm{NIH})$.

Histology and IHC. PIN lesions were graded using the nomenclature and criteria developed by Park et al. (54). In brief, HGPIN is characterized by intraglandular proliferation of crowding cells with atypia and cribriform formation or the development of multilayered solid glandular structures. Invasive adenocarcinoma is characterized by the proliferation of atypical cells that break the basal membrane and invade through the prostatic stroma. For metastasis studies, we performed CK8 or AR immunostaining in lymph nodes or lungs and recorded the percentage of mice that were positive. The quantitative results of tumor progression are from 2 random slides of each mouse for a total of 4-8 pairs of $\mathrm{PTEN}^{\mathrm{PC}-/-} \mathrm{PTEN}^{\mathrm{PC}-/-} \mathrm{WHSC1}^{\mathrm{PCOE} /+}$, and $\mathrm{PTEN}^{\mathrm{PC}-/-} \mathrm{WHSC1}^{\mathrm{PC}-/-}$ mice. The statistical significance between groups was determined by 2-tailed Fisher's exact test. All the primary antibodies used in this study were purchased from Cell Signaling Technology (antibodies directed against cyclin D1, 2926; Ki-67, 12202; phospho-AKT, 4060; phospho-S6, 2215; and RICTOR, 2114), with the exception of antibodies directed against AR (Santa Cruz Biotechnology, SC816), CK8 (Covance, MMS-162P), aSMA (Sigma-Aldrich, A5228), WHSC1 (Abcam, ab75359), Rac1 (Cytoskeleton, ARC03; Thermo Fisher Scientific, PA1-091), and E-cadherin (BD, 610181).

RNA-Seq, ChIP-Seq, and data analysis. Total RNA from 4 groups of PC3 cells with or without WHSC1 depletion was subjected to HiSeq RNA-Seq, performed by BGI Tech Solutions Co. Ltd, China. Transcriptome reads from RNA-Seq experiments were mapped to the reference genome (hg19) using the Bowtie tool. The gene expression level was quantified by a software package called RSEM. The list of significance was operated by setting of a $P$ value threshold at 0.05 ; the raw data can be downloaded as GSE84868. The differentially expressed genes were subsequently analyzed for the enrichment of biological themes using the clusterProfile package and Ingenuity Pathway Analysis program (http://www.ingenuity.com/index.html). For the ChIP-Seq assay, the chromatin was prepared, and ChIP-Seq analysis was then performed by Active Motif Inc. using an antibody against H3K36me2 (Millipore, 07-274). The 75-nt sequence reads generated by Illumina sequencing were mapped to the genome using the Burrows-Wheeler Aligner algorithm with default settings. Only reads that passed Illumina's purity filter, aligned with no more than 2 mismatches, and mapped uniquely to the genome were used in the subsequent analysis. The SICER cutoff for the false discovery rate after controlling for significance was $1^{-10}$. In total, 23,502 intervals (WT) and 16,120 intervals (WHSC1 knockdown) were identified over input control, and the raw data can be downloaded as GSE84869. The average profile for tag distributions was generated using ngsplot version 2.61. Motifs enriched in a region of interest were found using the SeqPos motif tool default with a $P$ value cutoff of 0.05 .

IHC analysis of human TMAs and IB analysis of PCa tissues. An Asian radical prostatectomy cohort was described previously (40). Briefly, tissue samples with the clinical parameters of 118 PCa patients who received radical prostatectomy were collected (Supplemental Table 2). None of these patients received adjuvant therapy until BCR. The use of pathological specimens, as well as the review of all the pertinent patient record, was approved by the institutional ethics review board, and informed consent was obtained from the patients. IHC analyses were performed using specific anti-WHSC1 (Abcam, ab75359) and anti-phospho-AKT (Cell Signaling Technology, 4060) antibodies. Samples were scanned using a Bliss automated slide scanner to generate high-resolution digital images. Protein expression was scored and quantified by pathologists (BD) blinded to the outcome of the cases. Basically, the quantified method is based on a multiplicative index of the average staining intensity ( 0 to 3 ) and extent of staining ( 0 to 3 ) in the cores yielding a 10-point staining index ranging from 0 (no staining) to 9 (strong staining).

Expression profile and correlation. Expression profiles of $\mathrm{Yu}$ et al. (GSE6919) (37), Grasso et al. (GSE35988) (38), and Taylor et al. (GSE21032) (4) were downloaded and are summarized in Supplemental Table 1. Box-and-whisker plots depict the median, first and third quartiles, and minimum and maximum. Statistical significance was assessed by 1-way ANOVA analysis. Correlation values between 2 genes were determined by iterating through each probe associated with gene $\mathrm{X}$ and comparing it with each probe associated with gene $\mathrm{Y}$. As each probe may represent a different transcript isoform, the maximum correlation score was selected to represent the isoforms with the greatest positive coexpression. Strength of association was determined by Pearson correlation coefficients.

Analysis of Rac1 and mTOR activity, PTEN loss, and WHSC1 signatures in human PCa patients. Analysis of human tumor data sets was carried out essentially as previously described $(11,38,55)$. The Rac1 gene signature was derived from the gene expression profile of the colon cancer cell line SW480 (GSE78093). Fold change greater than or equal to $1.5, P$ value less than 0.05 , and probes repressed (823 unique genes) and induced (760 unique genes) by Rac1 knockdown were used to calculate the $t$ score. The mTOR signature (defined using a fold change $\geq 2, P<0.05$ ) consisted of RAD001 inhibition, which induced 1,936 probes $(1,668$ mouse genes that were transformed to 1,409 human homeotic genes by Human and Mouse Homology with phenotype annotations) and repressed 549 probes (474 mouse genes; 427 human homeotic genes). The WHSC1 gene signature was derived from our own gene expression profile data set (GSE84868), which consisted of 960 upregulated and 1,270 downregulated unique human genes. To define the degree of gene signature manifestation within the profiles from an external human tumor data set (e.g., GSE21032), we used the previously described $t$ score metric $(11,55-57)$. For example, the $t$ score was defined for each external profile as the 2-sided $t$-statistic comparing the average of the WHSC1-induced genes with the average of the WHSC1-repressed genes (genes within the human tumor data set were first centered to SDs from the median of the primary tumor specimens). For a given data set, the $t$ score contrasted the patterns of the WHSC1-induced genes against those of the WHSC1repressed genes in order to derive a single value denoting the coordinate expression of the 2 gene sets.

Gene set enrichment analysis. Signature genes were compared using gene set enrichment analysis (GSEA) to identify the ranked list of genes affected by the expression level of WHSC1. Genes were ranked by "Differ_of_classes," and the permutation type was "gene set" and other sets using the GSEA default. The thresholds for inclusion were $P$ less than 0.05 and $q$ less than 0.25 .

Clinical outcome analysis. Affymetrix Human Exon 1.0 ST Array profiles and clinical information regarding the PCa patients $(n=140)$ from the Taylor et al. cohort (GSE21032) were used for analyses (4). 
The predictive values of WHSC1, PTEN, Rac1, and mTOR signatures univariately with BCR were analyzed using the Cox proportional hazards regression model. The HR and 95\% CI were computed. The Cox proportional hazards model analysis (Forward LR method) with the WHSC1, PTEN, PSA level, and Gleason score were used to estimate the coefficients of individual genes. The $K$-means clustering algorithm described previously (9) was used to stratify patient cohorts. C-statistics analysis was conducted using the R "survcomp" and "rms" package. The statistical procedures used in the analyses included a comparative step that used the paired $t$ test to compare the $C$-statistics of the models.

Statistics. All experiments were performed using 3-15 mice or 3 independent repeated experiments with cells. Unless otherwise indicated, the data in the figures are presented as the mean \pm SEM. Pearson correlation coefficients were used to evaluate the relationships between WHSC1 and gene expression. Cox proportional hazards regression model and multivariate Cox proportional hazards model analyses were performed with the statistical software SPSS 22.0. Statistical significance was determined by Student's $t$ test, 1-way ANOVA, 2-way ANOVA, Pearson correlation coefficients, log-rank test, Fisher's exact test, or $\chi^{2}$ test. For computing gene signature scores based on expression profiling data from human tumors, genes were first $z$-normalized to the SD from the median across the primary tumor samples, and the average of the $z$-normalized values for all the genes in the signature was used to represent the signature score for each sample profile. For all statistical tests, the 0.05 level of confidence (2-sided) was accepted for statistical significance.

Study approval. All animal experiments were performed in compliance with the Guide for the Care and Use of Laboratory Animals (National Academies Press, 2011) and were approved by the Institutional Biomedical Research Ethics Committee of the Shanghai Institutes for Biological Sciences, Chinese Academy of Sciences. All cell lines used here are available at ATCC.
Accession codes. RNA-Seq and ChIP-Seq data have been deposited at the Gene Expression Omnibus (GEO) database under accession numbers GSE84868 and GSE84869.

\section{Author contributions}

JQ and NL conceived and designed the experimental approach, performed experiments, and prepared the manuscript as senior authors. NL, WX, and HY designed and performed most of the experiments. NL and MJ contributed to the computational analysis for the gene signature and the statistical analysis. WX and BD performed TMA and pathology analyses. YD, YL, SK, TS, JR, QP, $\mathrm{XL}$, and PZ performed and GH, QL, WX, XW, and YW supervised a specific subset of experiments and analyses.

\section{Acknowledgments}

We thank Sophia Tsai and Ming-Jer Tsai for critical comments. Our research was supported by the National Key Basic Research Program (2016YFC0902202); the National Natural Science Foundation of China (31471281 and 81422030 to JQ, 81502440 to MJ, 81572536 to WX, 81672850 to BD); the Strategic Priority Research Program of the Chinese Academy of Sciences (XDB19000000); the Science and Technology Commission of Shanghai Municipality (14140901500); the State Key Laboratory of Medical Genomics, Collaborative Innovation Center of Systems Biomedicine; and Innovation Funds for Translational Research of Shanghai Jiao Tong University School of Medicine (15ZH4002). JQ is a scholar of the National 1000 Young Talents Program of China.

Address correspondence to: Jun Qin, Key Laboratory of Stem Cell Biology, CAS Center for Excellence in Molecular Cell Science, Institute of Health Sciences, Shanghai Institutes for Biological Sciences, Chinese Academy of Sciences, 320 Yue Yang Road, Shanghai 200031, China. Phone: 86.21.54923326; E-mail: qinjun@sibs.ac.cn.
1. American Cancer Society. Cancer Facts \& Figures 2016. Atlanta, Georgia, USA: American Cancer Society; 2016.

2. Shen MM, Abate-Shen C. Molecular genetics of prostate cancer: new prospects for old challenges. Genes Dev. 2010;24(18):1967-2000.

3. Sartor AO, et al. Evaluating localized prostate cancer and identifying candidates for focal therapy. Urology. 2008;72(6 suppl):S12-S24.

4. Taylor BS, et al. Integrative genomic profiling of human prostate cancer. Cancer Cell. 2010;18(1):11-22.

5. Tomlins SA, et al. Integrative molecular concept modeling of prostate cancer progression. Nat Genet. 2007;39(1):41-51.

6. Tomlins SA, et al. Recurrent fusion of TMPRSS2 and ETS transcription factor genes in prostate cancer. Science. 2005;310(5748):644-648.

7. Robinson $\mathrm{D}$, et al. Integrative clinical genomics of advanced prostate cancer. Cell. 2015;161(5):1215-1228.

8. Wang S, et al. Prostate-specific deletion of the murine Pten tumor suppressor gene leads to metastatic prostate cancer. Cancer Cell. 2003;4(3):209-221.

9. Ding Z, et al. SMAD4-dependent barrier con- strains prostate cancer growth and metastatic progression. Nature. 2011;470(7333):269-273.

10. Chen Z, et al. Crucial role of p53-dependent cellular senescence in suppression of Pten-deficient tumorigenesis. Nature. 2005;436(7051):725-730.

11. Qin J, et al. COUP-TFII inhibits TGF- $\beta$-induced growth barrier to promote prostate tumorigenesis. Nature. 2013;493(7431):236-240.

12. Abate-Shen C, et al. Nkx3.1; Pten mutant mice develop invasive prostate adenocarcinoma and lymph node metastases. Cancer Res. 2003;63(14):3886-3890.

13. Carver BS, et al. Aberrant ERG expression cooperates with loss of PTEN to promote cancer progression in the prostate. Nat Genet. 2009;41(5):619-624.

14. Chen M, et al. Identification of PHLPP1 as a tumor suppressor reveals the role of feedback activation in PTEN-mutant prostate cancer progression. Cancer Cell. 2011;20(2):173-186.

15. King JC, et al. Cooperativity of TMPRSS2-ERG with PI3-kinase pathway activation in prostate oncogenesis. Nat Genet. 2009;41(5):524-526.

16. Lei Q, et al. NKX3.1 stabilizes p53, inhibits AKT activation, and blocks prostate cancer initiation caused by PTEN loss. Cancer Cell.
2006;9(5):367-378

17. Mulholland DJ, et al. Pten loss and RAS/MAPK activation cooperate to promote EMT and metastasis initiated from prostate cancer stem/progenitor cells. Cancer Res. 2012;72(7):1878-1889.

18. Baylin SB, Jones PA. A decade of exploring the cancer epigenome - biological and translational implications. Nat Rev Cancer. 2011;11(10):726-734.

19. Albert M, Helin K. Histone methyltransferases in cancer. Semin Cell Dev Biol. 2010;21(2):209-220.

20. McCabe MT, et al. EZH2 inhibition as a therapeutic strategy for lymphoma with EZH2-activating mutations. Nature. 2012;492(7427):108-112.

21. Knutson SK, et al. Durable tumor regression in genetically altered malignant rhabdoid tumors by inhibition of methyltransferase EZH2. Proc Natl Acad Sci U S A. 2013;110(19):7922-7927.

22. Min J, et al. An oncogene-tumor suppressor cascade drives metastatic prostate cancer by coordinately activating Ras and nuclear factor- $\mathrm{\kappa B}$. Nat Med. 2010;16(3):286-294.

23. Varambally S, et al. The polycomb group protein $\mathrm{EZH} 2$ is involved in progression of prostate cancer. Nature. 2002;419(6907):624-629.

24. Asangani IA, et al. Characterization of the EZH2MMSET histone methyltransferase regulatory 
axis in cancer. Mol Cell. 2013;49(1):80-93.

25. Kuo AJ, et al. NSD2 links dimethylation of histone $\mathrm{H} 3$ at lysine 36 to oncogenic programming. Mol Cell. 2011;44(4):609-620.

26. Li Y, et al. The target of the NSD family of histone lysine methyltransferases depends on the nature of the substrate. J Biol Chem. 2009;284(49):34283-34295.

27. Stec I, et al. WHSC1, a 90 kb SET domain-containing gene, expressed in early development and homologous to a Drosophila dysmorphy gene maps in the Wolf-Hirschhorn syndrome critical region and is fused to IgH in $\mathrm{t}(4 ; 14)$ multiple myeloma. Hum Mol Genet. 1998;7(7):1071-1082.

28. Nimura K, et al. A histone $\mathrm{H} 3$ lysine 36 trimethyltransferase links Nkx2-5 to Wolf-Hirschhorn syndrome. Nature. 2009;460(7252):287-291.

29. Keats JJ, et al. Overexpression of transcripts originating from the MMSET locus characterizes all $\mathrm{t}(4 ; 14)$ (p16;q32)-positive multiple myeloma patients. Blood. 2005;105(10):4060-4069.

30. Santra M, Zhan F, Tian E, Barlogie B, Shaughnessy J. A subset of multiple myeloma harboring the $t(4 ; 14)(\mathrm{p} 16 ; \mathrm{q} 32)$ translocation lacks FGFR3 expression but maintains anIGH/MMSET fusion transcript. Blood. 2003;101(6):2374-2376.

31. Chesi M, Nardini E, Lim RSC, Smith KD, Kuehl WM, Bergsagel PL. The t $(4 ; 14)$ translocation in myeloma dysregulates both FGFR3and a novel gene, MMSET, resulting in IgH/MMSET hybrid transcripts. Blood.1998;92(9):3025-3034.

32. Hudlebusch HR, et al. The histone methyltransferase and putative oncoprotein MMSET is overexpressed in a large variety of human tumors. Clin Cancer Res. 2011;17(9):2919-2933.

33. Teresa E-I, et al. The histone methyl transferase MMSET/WHSC1 promotes an epithelial-mesenchymal transition and invasive properties of prostate cancer. Cancer Res. 2012;72(8 supplement):1046.

34. Saloura V, et al. WHSC1 promotes oncogenesis through regulation of NIMA-related kinase-7 in squamous cell carcinoma of the head and neck.
Mol Cancer Res. 2015;13(2):293-304.

35. Ezponda T, et al. The histone methyltransferase MMSET/WHSC1 activates TWIST1 to promote an epithelial-mesenchymal transition and invasive properties of prostate cancer. Oncogene. 2013;32(23):2882-2890.

36. Yang $\mathrm{P}$, et al. Histone methyltransferase NSD2/ MMSET mediates constitutive NF- $\kappa \mathrm{B}$ signaling for cancer cell proliferation, survival, and tumor growth via a feed-forward loop. Mol Cell Biol. 2012;32(15):3121-3131.

37. Yu YP, et al. Gene expression alterations in prostate cancer predicting tumor aggression and preceding development of malignancy. J Clin Oncol. 2004;22(14):2790-2799.

38. Grasso CS, et al. The mutational landscape of lethal castration-resistant prostate cancer. Nature. 2012;487(7406):239-243.

39. Gulzar ZG, McKenney JK, Brooks JD. Increased expression of NuSAP in recurrent prostate cancer is mediated by E2F1. Oncogene. 2013;32(1):70-77.

40. Xu F, et al. Decreased TSPAN1 promotes prostate cancer progression and is a marker for early biochemical recurrence after radical prostatectomy. Oncotarget. 2016;7(39):63294-63305.

41. Chen ML, et al. The deficiency of Akt1 is sufficient to suppress tumor development in $\mathrm{Pten}^{+/}$ mice. Genes Dev. 2006;20(12):1569-1574.

42. Shen MM, Abate-Shen C. Pten inactivation and the emergence of androgen-independent prostate cancer. Cancer Res. 2007;67(14):6535-6538.

43. Li X, Liu J, Gao T. $\beta$-TrCP-mediated ubiquitination and degradation of PHLPP1 are negatively regulated by Akt. Mol Cell Biol. 2009;29(23):6192-6205.

44. Li Q, et al. Akt phosphorylates Wnt coactivator and chromatin effector Pygo 2 at serine 48 to antagonize its ubiquitin/proteasome-mediated degradation. J Biol Chem. 2015;290(35):21553-21567.

45. Gao D, Inuzuka H, Tseng A, Chin RY, Toker A, Wei W. Phosphorylation by Akt1 promotes cytoplasmic localization of Skp2 and impairs APC-
Cdh1-mediated Skp2 destruction. Nat Cell Biol. 2009;11(4):397-408.

46. Terai K, Abbas T, Jazaeri AA, Dutta A. CRL4Cdt2 E3 ubiquitin ligase monoubiquitinates PCNA to promote translesion DNA synthesis. Mol Cell. 2010;37(1):143.

47. Jackson S, Xiong Y. CRL4s: the CUL4-RING E3 ubiquitin ligases. Trends Biochem Sci. 2009;34(11):562-570.

48. Sahai E, Marshall CJ. RHO-GTPases and cancer. Nat Rev Cancer. 2002;2(2):133-142.

49. Evans DL, et al. MMSET is dynamically regulated during cell-cycle progression and promotes normal DNA replication. Cell Cycle. 2016;15(1):95-105.

50. Ernst T, et al. Inactivating mutations of the his tone methyltransferase gene $\mathrm{EZH} 2$ in myeloid disorders. Nat Genet. 2010;42(8):722-726.

51. Ko M, et al. Impaired hydroxylation of 5-methylcytosine in myeloid cancers with mutant TET2. Nature. 2010;468(7325):839-843.

52. Turcan S, et al. IDH1 mutation is sufficient to establish the glioma hypermethylator phenotype. Nature. 2012;483(7390):479-483.

53. Jin C, McKeehan K, Wang F. Transgenic mouse with high Cre recombinase activity in all prostate lobes, seminal vesicle, and ductus deferens. Prostate. 2003;57(2):160-164.

54. Park JH, et al. Prostatic intraepithelial neoplasia in genetically engineered mice. Am J Pathol. 2002;161(2):727-735.

55. Qin J, et al. Androgen deprivation-induced NCoA2 promotes metastatic and castration-resistant prostate cancer. J Clin Invest. 2014;124(11):5013-5026.

56. Creighton CJ, et al. Integrated analyses of microRNAs demonstrate their widespread influence on gene expression in high-grade serous ovarian carcinoma. PLoS One. 2012;7(3):e34546.

57. Cancer Genome Atlas Research Network. Comprehensive molecular characterization of clear cell renal cell carcinoma. Nature. 2013;499(7456):43-49. 